\title{
Variação primeira e segunda para o primeiro autovalor de um problema elíptico
}

\author{
Sérgio Tadao Martins
}

\author{
DisSERTAÇÃO APRESENTADA \\ $\mathrm{AO}$ \\ Instituto DE MATEMÁticA E Estatística \\ DA \\ Universidade DE SÃo PAUlo \\ PARA \\ OBTENÇÃO DO TÍTULO \\ Mestre EM CiÊNCIAS
}

Área de Concentração: Matemática
Orientador: Prof. Dr. Paolo Piccione

Durante o desenvolvimento deste trabalho o autor recebeu auxílio financeiro da FAPESP

São Paulo, novembro de 2007 



\title{
Variação primeira e segunda para o primeiro autovalor de um problema elíptico
}

\author{
Este exemplar corresponde à redação \\ final da dissertação devidamente corrigida \\ e defendida por Sérgio Tadao Martins \\ e aprovada pela Comissão Julgadora.
}

Banca Examinadora:

- Prof. Dr. Paolo Piccione (orientador) - IME-USP.

- Prof. Dr. Antônio Luiz Pereira - IME-USP.

- Prof. Dr. Francesco Mercuri - UNICAMP. 



\section{Resumo}

Consideraremos o problema elíptico $-\Delta u+\alpha \chi_{D} u=\lambda u$ em $\Omega$, onde $\Omega$ é um dominio de $\mathbb{R}^{n}$ com fronteira regular, e $D \subset \Omega$ é um subconjunto fechado de medida de Lebesgue fixada. A motivação para este problema vem da Mecânica, onde esta equação é encontrada no estudo de vibrações de uma mebrana composta. Seja $\lambda_{1}(D)$ o primeiro autovalor do problema, como função do conjunto $D$. Nesse trabalho mostraremos que $\lambda_{1}$ é um autovalor simples, e estudaremos o problema de minimizar $\lambda_{1}$ ao variarmos $D$ no conjunto de todos os subconjuntos de medida fixada de $\Omega$. Mais especificamente, determinaremos fórmulas para a variação primeira e segunda de $\lambda_{1}$.

Palavras-chave: membrana composta, problemas elípticos, Laplaciano, teorema espectral 


\section{Abstract}

We will consider the elliptic problem $-\Delta u+\alpha \chi_{D} u=\lambda u$ in $\Omega$, where $\Omega$ is a domain in $\mathbb{R}^{n}$ with regular boundary, and $D \subset \Omega$ is a closed subset with prescribed Lebesgue measure. The motivation for this problem comes from Mechanics, where this equation models the vibrations of a composite membrane. Let $\lambda_{1}(D)$ be the first eigenvalue of the problem, which is seen as a function of the set $D$. In this work, we will show that $\lambda_{1}$ is a simple eigenvalue, and we will study the problem of minimizing $\lambda_{1}(D)$ when $D$ varies in the family of all closed subsets of $\Omega$ with a given Lebesgue measure. More precisely, we will determine formulas for the first and the second variation of $\lambda_{1}$.

Keywords: composite membrane, elliptic problem, Laplacian, spectral theorem 


\section{Sumário}

1 Introdução 1

2 Operadores Compactos e de Fredholm 5

2.1 Espaços Métricos Compactos . . . . . . . . . . . . . . . . . 5

2.2 Operadores Compactos . . . . . . . . . . . . . . . . . . . . 10

2.3 Operadores de Fredholm . . . . . . . . . . . . . . . . . . . . . 12

2.4 Teorema Espectral . . . . . . . . . . . . . . . . . . . . . . . 17

3 Os espaços $L^{p}$ e os espaços de Sobolev 23

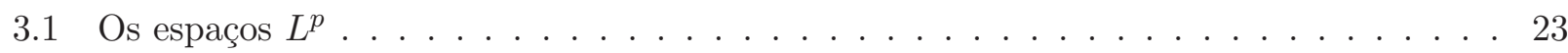

3.2 Os espaços de Sobolev . . . . . . . . . . . . . . . . . . . . . . . 28

4 O operador $-\Delta+\alpha \chi_{\mathrm{D}} \quad 37$

4.1 Membranas compostas ótimas . . . . . . . . . . . . . . . . . . . 37

4.2 Estudo do operador $-\Delta+\alpha \chi_{D} \ldots \ldots \ldots \ldots \ldots \ldots$

5 Fórmulas de variação para o primeiro autovalor $\quad 51$

5.1 Primeiro método: primeira variação . . . . . . . . . . . . . . . . . 52

5.2 Segundo método: primeira e segunda variações . . . . . . . . . . . . . . . 56

5.3 Perspectivas futuras . . . . . . . . . . . . . . . . . . . 62

Referências Bibliográficas $\quad 65$ 


\section{Capítulo 1}

\section{Introdução}

O estudo de membranas vibrantes é um assunto clássico em Física-Matemática que originou muitos avanços na teoria de equações diferenciais parciais elípticas. Entre os tópicos mais estudados dentro deste assunto e um dos mais importantes é aquele que se refere à estimativas dos harmônicos naturais de uma membrana vibrante.

Uma membrana vibrante, com distribuição de massa homogênea, é descrita matematicamente por um conjunto $\Omega \in \mathbb{R}^{n}$ e pela equação

$$
\Delta f=\frac{\partial^{2} f}{\partial t^{2}}
$$

em que $f(x, t)$ depende da posição $x \in \mathbb{R}^{n}$ e de $t \in \mathbb{R}$, que é interpretado como a variável tempo. As condições inicial e de fronteira são supostas dadas de antemão. O conjunto $\Omega$ representa a forma que a membrana ocupa em repouso, e em geral é um conjunto aberto cuja fronteira $\partial \Omega$ é suficientemente regular. A forma da membrana no instante $t$ é dada pelo gráfico da função $f$ sobre $\Omega$. Duas boas referências para este assunto são [8] e [6].

Para resolver a equação (1.1), utilizamos a técnica de separação de variáveis, isto é, procuramos soluções da forma

$$
f(x, t)=u(x) v(t)
$$

Substituindo diretamente em (1.1), obtemos

$$
\frac{\Delta u}{u}=\frac{v^{\prime \prime}}{v}
$$

Observando que o lado esquerdo depende somente de $x$ e o lado direito somente de $t$, concluimos que 
ambos os lados devem ser iguais a uma constante, o que nos leva ao problema do autovalor para o Laplaciano

$$
-\Delta u=\lambda u \quad \text { em } \Omega
$$

em que o sinal negativo é colocado apenas para tornar o autovalor $\lambda$ não negativo, ou até mesmo estritamente positivo em alguns casos importantes.

É bastante comum que tenhamos também uma condição de fronteira vinda do problema original. Se a membrana está fixa na fronteira de $\Omega$, ou seja, se $f(x, t)=0$ sempre que $x \in \partial \Omega$ para todo $t \in \mathbb{R}$, então também teremos $u(x)=0$ para todo $x \in \mathbb{R}^{n}$, condição que é conhecida como condição de fronteira de Dirichlet (descartamos o caso em que $v(t)=0$ para todo $t \in \mathbb{R}$, o que nos dá a solução na qual a membrana permanece em repouso), que nos leva ao problema

$$
\left\{\begin{array}{l}
-\Delta u=\lambda u, \quad \text { em } \Omega \\
u=0, \quad \text { em } \partial \Omega .
\end{array}\right.
$$

Uma solução não trivial $u$ do problema acima é chamada de autofunção (de Dirichlet) do Laplaciano, e $\lambda$ é chamado de autovalor (de Dirichlet) do Laplaciano. O resultado fundamental é o seguinte Teorema Espectral para o Laplaciano: se $\Omega \in \mathbb{R}^{n}$ é um aberto conexo e limitado, então existe um conjunto discreto ilimitado de autovalores

$$
0<\lambda_{1}<\lambda_{2} \leq \lambda_{3} \leq \ldots
$$

do Laplaciano, e uma seqüência $\left(u_{k}\right)_{k \geq 1}$ de autofunções associadas, as quais podem ser tomadas duas a duas ortogonais e com norma igual a $1 \mathrm{em} L^{2}(\Omega)$. Pode-se demonstrar também que as funções $u_{k}$ são analíticas em $\Omega$ e $u_{1}$ pode ser tomada positiva em $\Omega$. Além disso, o autovalor $\lambda_{1}$ é simples (por isso a desigualdade estrita $\lambda_{1}<\lambda_{2}$ ).

J. W. S. Rayleigh [17] estudou o valor de $\lambda_{1}$ em função de $\Omega$, no caso bidimensional, supondo a área de $\Omega$ fixa. Ele observou que $\lambda_{1}$ era o menor possível quando $\Omega$ era um disco, e conjecturou essa propriedade minimizante como algo que caracterizaria o disco. Esta conjectura foi demonstrada, independentemente, por Faber [10] e Krahn [14], e ficou conhecida como desigualdade de Faber-Krahn.

Neste trabalho, consideraremos o caso em que a membrana vibrante não é homogênea, isto é, a função densidade de massa $\rho: \Omega \rightarrow \mathbb{R}$ não é constante. Novamente, podemos usar o método de 
separação de variáveis para obter o seguinte problema de autovalor:

$$
\left\{\begin{array}{l}
-\Delta u=\lambda \rho u, \quad \text { em } \Omega \\
u=0, \quad \text { em } \partial \Omega .
\end{array}\right.
$$

O Teorema Espectral pode ser estendido para esse novo problema e, nesta nova situação, podemos propor um problema similar ao estudado por Rayleigh. Supomos, em primeiro lugar, que a densidade está limitada entre dois valores, isto é, existem $\rho_{1}$ e $\rho_{2}$ tais que $0<\rho_{1} \leq \rho(x) \leq \rho_{2}<+\infty$ para todo $x \in \Omega$. Esta suposição, do ponto de vista físico, é bastante natural. Em seguida, fixamos a massa total $M$ da membrana,

$$
M=\int_{\Omega} \rho d x
$$

e perguntamos qual distribuição de densidade minimiza o primeiro autovalor $\lambda_{1}$. O resultado surpreendente que se mostra é que a distribuição $\rho$ que minimiza $\lambda_{1}$ não é contínua. Mais especificamente, a membrana que possui $\lambda_{1}$ mínimo é composta de dois materiais diferentes, um com densidade $\rho_{1}$, e outro com densidade $\rho_{2}$.

Esta membrana ótima formada por dois materiais de densidades distintas é o que se conhece como membrana composta. Podemos então considerar as membranas que já possuem este tipo de distribuição de massa e estudar problemas relacionados aos seus autovalores. A formulação matemática é a seguinte: seja $D$ um subconjunto fechado de $\Omega$ e considere a função densidade $\rho=\rho_{1} \chi_{D}+\rho_{2} \chi_{D^{c}}$. Fixar a massa $M$ da membrana significa que a medida de $D$ está fixada, e pode-se mostrar que o problema de autovalor que obtemos é

$$
(*)_{\Omega, \alpha, D}=\left\{\begin{array}{l}
-\Delta u+\alpha \chi_{D} u=\lambda u \text { em } \Omega \\
u=0 \text { em } \partial \Omega,
\end{array}\right.
$$

no qual a constante $\alpha>0$ depende de $\rho_{1}, \rho_{2}$ e da área de $D$. Para este novo problema, também é válido o Teorema Espectral. De fato, mostraremos que o inverso do operador $-\Delta+\alpha \chi_{D}$ é compacto e auto-adjunto. Podemos então considerar o seguinte problema variacional para o primeiro autovalor: fixados $\Omega, \alpha>0$ e $|D|$, queremos determinar o conjunto $D$ que minimiza o primeiro autovalor $\lambda_{1}$ de $(*)_{\Omega, \alpha, D}$.

No nosso trabalho temos como objetivo o cálculo da variação primeira e segunda do autovalor $\lambda_{1}$ como função de $\Omega$. Existem dois tipos de dificuldades a serem enfrentadas no desenvolvimento das computações: 
(a) o conjunto onde o problema variacional está definido, que consiste de todos os subconjuntos $D \subset \Omega$ mensuráveis e com volume fixado, não é um espaço de Banach, ou, mais geralmente, não possui uma estrutura de variedade;

(b) os dados do problema variacional têm baixa regularidade, e uma abordagem rigorosa ao problema envolveria técnicas sofisticadas de teoria geométrica da medida.

Na nossa dissertação não entraremos nos detalhes do problema (b).

Quanto às técnicas usadas para o cálculo das variações primeira e segunda, a nossa referência principal é o trabalho [4], de onde são tomadas as principais idéias dessa dissertação. Para o cálculo da variação primeira, consideraremos uma curva $\varphi_{t}: \Omega \rightarrow \Omega$ que preserva o volume e tais que $\varphi_{0}=\operatorname{Id}_{\Omega}$. As fórmulas desejadas são obtidas diferenciando o problema em relação à variável $t$, utilizando funções regulares aproximantes das funções caraterísticas, e o teorema da Divergência.

O trabalho está estruturado da seguinte maneira: o Capítulo 2 contém a teoria principal a respeito dos operadores compactos e dos operadores de Fredholm, e é concluído com o Teorema Espectral para operadores compactos auto-adjuntos. O Capítulo 3 recorda as principais propriedades dos espaços $L^{p}$ e dos espaços de Sobolev. Neste capítulo, são apresentadas desigualdades importantes e que usaremos repetidas vezes, como a desigualdade de Hölder, a desigualdade de Sobolev e a desigualdade de Poincaré, bem como o Teorema de Compacidade de Rellich-Kondrachov e o fato que $C_{0}^{\infty}(\Omega)$ é denso em $L^{p}(\Omega)$ para $\Omega \in \mathbb{R}^{n}$ aberto e $1 \leq p<\infty$. O Capítulo 4 fornece detalhes a respeito do problema das membranas compostas e estende o Teorema Espectral para o operador $-\Delta+\alpha \chi_{D}$, supondo $\alpha>0$ e $D$ fixados. Finalmente, o Capítulo 5 apresenta as fórmulas de primeira e segunda variação para o primeiro autovalor de $(*)_{\Omega, \alpha, D}$ quando variamos o conjunto $D$. 


\section{Capítulo 2}

\section{Operadores Compactos e de Fredholm}

\subsection{Espaços Métricos Compactos}

Iniciamos recordando brevemente alguns fatos fundamentais a respeito de espaços métricos compactos.

Proposição 2.1 Um subconjunto seqüencialmente compacto de um espaço métrico é fechado, limitado e separável.

Demonstração: Seja $K$ um subconjunto seqüencialmente compacto do espaço métrico $(X, d)$.

Seja $\left(x_{n}\right)_{n \geq 1}$ uma seqüência em $K$ que converge para $y \in X$. Pelo enunciado, $\left(x_{n}\right)_{n \geq 1}$ possui uma subseqüência $\left(x_{m_{n}}\right)_{n \geq 1}$ que converge para $y^{\prime} \in K$. Mas é claro que $y^{\prime}=y$, logo $y \in K$ e $K$ é fechado.

Suponha agora que $K$ não é limitado. Então existe uma seqüência $\left(x_{n}\right)_{n \geq 1}$ tal que $d\left(x_{n}, z\right) \rightarrow \infty$ quando $n \rightarrow \infty$, em que $z$ é qualquer ponto fixado. Mas então, para todo $y$ e para toda subseqüência $\left(x_{m_{n}}\right)_{n \geq 1}$ de $\left(x_{n}\right)_{n \geq 1}$, temos $d\left(x_{m_{n}}, y\right) \rightarrow \infty$ quando $n \rightarrow \infty$, e portanto $\left(x_{n}\right)_{n \geq 1}$ não possui uma subseqüência convergente, uma contradição. Logo $K$ é limitado.

Finalmente, mostremos que $K$ é separável. Tome qualquer $x_{0} \in K$ e seja $d_{0}=\sup _{x \in K} d\left(x_{0}, x\right)$. Como $K$ é limitado, $d_{0}<\infty$. Escolhemos agora $x_{1} \in K$ tal que $d\left(x_{0}, x_{1}\right) \geq d_{0} / 2$ e, recursivamente, escolhemos $x_{n+1} \in K$ tal que $\min _{0 \leq j \leq n} d\left(x_{j}, x_{n+1}\right) \geq d_{n} / 2$, em que $d_{n}=\sup _{x \in K} \operatorname{mín}_{0 \leq j \leq n} d\left(x_{j}, x\right)$. Claramente $d_{0} \geq d_{1} \geq d_{2} \geq \ldots$. Se $d_{n} \geq \delta>0$ para todo $n$, então nenhuma subseqüência de $\left(x_{n}\right)_{n \geq 0}$ é uma seqüência de Cauchy, contradizendo a hipótese de que $K$ é seqüencialmente compacto. Portanto 
$d_{n} \rightarrow 0$. Mas, se $d_{n} \rightarrow 0$, então o conjunto $\left\{x_{n}: n \geq 0\right\}$ é denso em $K$, demonstrando que $K$ é separável.

Proposição 2.2 Um subconjunto de um espaço métrico é compacto se, e somente se, é seqüencialmente compacto.

Demonstração: Seja $K$ um subconjunto compacto do espaço métrico $X$. Se $K$ não é seqüencialmente compacto, então existe uma seqüência $\left(x_{n}\right)_{n \geq 1}$ em $K$ que não possui pontos de acumulação em $K$. Esta seqüência contém uma subseqüência infinita cujos elementos são dois a dois distintos. Denotemos esta subseqüência por $\left(y_{n}\right)_{n \geq 1}$. Para cada $n \geq 1$, existe uma bola aberta $B\left(y_{n}, r_{n}\right)$ tal que $B\left(y_{n}, r_{n}\right) \cap K$ não contém nenhum ponto $y_{m}$ com $m \neq n$. Os conjuntos $B\left(y_{n}, r_{n}\right)$, para $n \geq 1$, junto com o conjunto $K-\left\{y_{1}, y_{2}, \ldots\right\}$, formam uma cobertura de $K$ para a qual não existe subcobertura finita, contradizendo o fato de $K$ ser compacto. Logo $K$ deve ser seqüencialmente compacto.

Reciprocamente, suponha que $K$ é seqüencialmente compacto. Em primeiro lugar, mostraremos que toda cobertura enumerável de $K$ por abertos de $X$ possui uma subcobertura finita. Sejam $E_{1}$, $E_{2}, E_{3}, \ldots$ os abertos de uma cobertura de $K$. Suponha que, para todo $n, \bigcup_{j=1}^{n} E_{j}$ não cobre $K$. Então existe uma seqüência $\left(x_{n}\right)_{n \geq 1}$ em $K$ tal que $x_{n} \notin \bigcup_{j=1}^{n} E_{j}$. Seja $\left(x_{m_{n}}\right)_{n \geq 1}$ uma subseqüência de $\left(x_{n}\right)_{n \geq 1}$ que converge para um ponto $y \in K$. Como $X-\bigcup_{j=1}^{n} E_{j}$ é um conjunto fechado que contém todos os pontos $x_{m} \operatorname{com} m \geq n, y$ deve pertencer a este conjunto. Mas então $y \in\left(X-\bigcup_{j=1}^{\infty} E_{j}\right)$, isto é, $y \notin \bigcup_{j=1}^{\infty} E_{j}$. Logo $y \notin K$, o que é impossível.

Seja agora $\left\{E_{\alpha}\right\}$ uma cobertura qualquer de $K$ por abertos de $X$. Sabemos que $K$ é separável. Seja então $\left\{y_{1}, y_{2}, \ldots\right\}$ um subconjunto denso de $K$. O conjunto de bolas

$$
\mathcal{B}=\left\{B\left(y_{n}, m^{-1}\right): n, m \geq 1\right\}
$$

tem a seguinte propriedade: para todo aberto $G \subset X$, o conjunto $G \cap K$ é a união de conjuntos da forma $K \cap B\left(y_{\tilde{n}}, \tilde{m}^{-1}\right)$, com $B\left(y_{\tilde{n}}, \tilde{m}^{-1}\right) \in \mathcal{B}$.

Sejam $B_{1}, B_{2}, B_{3}, \ldots$ as bolas $B\left(y_{\tilde{n}}, \tilde{m}^{-1}\right)$ que ocorrem quando $G$ varia sobre os conjuntos $E_{\alpha}$. Cada $B_{j}$ está contida em um dos conjuntos $E_{\alpha_{j}}$. Logo os conjuntos $E_{\alpha_{1}}, E_{\alpha_{2}}, E_{\alpha_{3}}, \ldots$ formam uma cobertura aberta enumerável de $K$, e o resultado segue. 
Corolário 2.1 Um subconjunto compacto de um espaço métrico é fechado, limitado e separável.

Definição 2.1 Um subconjunto $K$ do espaço métrico $X$ é totalmente limitado se para todo $\varepsilon>0$ existe um número finito de bolas $B\left(x_{i}, \varepsilon\right)$ com $x_{i} \in X$ que cobrem $K$. Tais bolas constituem o que chamamos de uma $\varepsilon$-cobertura.

Teorema 2.1 Seja $X$ um espaço métrico completo. Um subconjunto fechado $K \subset X$ é compacto se, e somente se, é totalmente limitado.

Demonstração: Suponha que $K$ é compacto. Para todo $\varepsilon>0$, o conjunto de bolas $\{B(x, \varepsilon)$ : $x \in K\}$ constitui uma cobertura aberta de $K$, a qual possui uma subcobertura finita. Logo $K$ é totalmente limitado.

Reciprocamente, seja $K$ fechado e totalmente limitado. Mostraremos que $K$ é seqüencialmente compacto. Seja $\left(y_{n}\right)_{n \geq 1}$ uma seqüência em $K$. Considere uma 1-cobertura $\left\{B\left(x_{1}, 1\right), \ldots, B\left(x_{m}, 1\right)\right\}$ de $K$. Pelo menos uma das bolas $B\left(x_{i}, 1\right)$ contém uma subseqüência infinita $\left(y_{n}^{1}\right)_{n \geq 1}$ de $\left(y_{n}\right)_{n \geq 1}$. Em seguida, considere uma $\frac{1}{2}$-cobertura de $K$ por um número finito de bolas, e extraia uma subseqüência $\left(y_{n}^{2}\right)_{n \geq 1}$ de $\left(y_{n}^{1}\right)_{n \geq 1}$ que esteja completamente contida em uma das bolas de raio $\frac{1}{2}$. Procedendo recursivamente, extraímos uma subseqüência $\left(y_{n}^{m}\right)_{n \geq 1}$ de $\left(y_{n}^{m-1}\right)_{n \geq 1}$ que esteja contida numa bola de raio $m^{-1}$. Desse modo, a seqüência $\left(y_{n}^{n}\right)_{n \geq 1}$ é uma seqüência de Cauchy. Como $X$ é completo, existe $\lim _{n \rightarrow \infty} y_{n}^{n}$ em $X$. Este limite também pertence a $K$, pois $K$ é fechado. Portanto $K$ é seqüencialmente compacto.

Definição 2.2 Uma família $\left\{f_{\alpha}\right\}$ de funções definidas em um conjunto $Y$ é dita uniformemente limitada se existe uma constante $C$ tal que $\left|f_{\alpha}(x)\right| \leq C$ para todo $x \in Y$ e para todo $\alpha$.

Definição 2.3 Uma familia $\left\{f_{\alpha}\right\}$ de funções contínuas definidas em um subconjunto $Y$ do espaço métrico $(X, d)$ é dita eqüicontínua se para todo $\varepsilon>0$ existe $\delta>0$ tal que

$$
d(x, y)<\delta \Longrightarrow\left|f_{\alpha}(x)-f_{\alpha}(y)\right|<\varepsilon
$$

para quaisquer $x, y \in Y$ e para todo $\alpha$. 
Lema 2.1 Seja $\mathcal{H}$ uma família de funções uniformemente limitada e eqüicontínua no espaço métrico compacto $(X, d)$. Então toda seqüência $\left(f_{n}\right)_{n \geq 1}$ de funções em $\mathcal{H}$ possui uma subseqüência que converge uniformemente em $X$ para uma função contínua.

Demonstração: Seja $\left(x_{n}\right)_{n \geq 1}$ uma seqüência densa em $X$. Como a seqüência $\left(f_{n}\left(x_{1}\right)\right)_{n \geq 1}$ é limitada, existe uma subseqüência $\left(f_{n}^{1}\left(x_{1}\right)\right)_{n \geq 1}$ convergente. A seqüência $\left(f_{n}^{1}\left(x_{2}\right)\right)_{n \geq 1}$ é limitada, logo possui uma subseqüência $\left(f_{n}^{2}\left(x_{2}\right)\right)_{n \geq 1}$ convergente. Procedendo deste modo, extraímos uma subseqüência $\left(f_{n}^{k}\left(x_{k}\right)\right)_{n \geq 1}$ convergente da seqüência $\left(f_{n}^{k-1}\left(x_{k}\right)\right)_{n \geq 1}$. Seja $g_{n}=f_{n}^{n}$. Temos que $\left(g_{n}\left(x_{k}\right)\right)_{n \geq 1}$ é convergente para todo $k$. Provaremos que $\left(g_{n}\right)_{n \geq 1}$ é uniformemente convergente em $X$. Como a família $\left\{g_{n}\right\}$ é eqüicontínua, para todo $\varepsilon>0$ existe um $\delta>0$ tal que

$$
\left|g_{n}(x)-g_{n}(y)\right|<\varepsilon \quad \text { sempre que } \quad d(x, y)<\delta,
$$

para todos $x, y \in X$ e $n \geq 1$. Para todo $x \in X$ escrevemos

$$
\left|g_{n}(x)-g_{m}(x)\right| \leq\left|g_{n}(x)-g_{n}\left(x_{k}\right)\right|+\left|g_{n}\left(x_{k}\right)-g_{m}\left(x_{k}\right)\right|+\left|g_{m}\left(x_{k}\right)-g_{m}(x)\right|,
$$

em que $x_{k}$ é tal que $d\left(x, x_{k}\right)<\delta$. Então

$$
\left|g_{n}(x)-g_{m}(x)\right|<2 \varepsilon+\left|g_{n}\left(x_{k}\right)-g_{m}\left(x_{k}\right)\right| .
$$

Afirmamos que existe um número finito de pontos $x_{1}, x_{2}, \ldots, x_{\ell}$, tais que para todo $x \in X$ existe um ponto $x_{k}$, com $1 \leq k \leq \ell$ tal que $d\left(x, x_{k}\right)<\delta$. De fato, tome uma $(\delta / 2)$-cobertura finita de $X$ por bolas $B_{1}, B_{2}, \ldots, B_{p}$ e escolha em cada bola $B_{j}$ um ponto $x_{\alpha_{j}}$ da seqüência $\left(x_{n}\right)_{n \geq 1}$. Basta então tomar $\ell=\operatorname{máx}\left\{\alpha_{1}, \ldots, \alpha_{p}\right\}$.

Para cada $k, 1 \leq k \leq \ell$, existe um inteiro positivo $n_{k}$ tal que

$$
\left|g_{n}\left(x_{k}\right)-g_{m}\left(x_{k}\right)\right|<\varepsilon \quad \text { se } \quad m \geq n \geq n_{k} .
$$

Usando essa relação em (2.1), obtemos $\left|g_{n}(x)-g_{m}(x)\right|<3 \varepsilon$ se $m \geq n \geq \operatorname{máx}\left\{n_{1}, n_{2}, \ldots, n_{\ell}\right\}$. Logo $\left(g_{n}\right)_{n \geq 1}$ é uniformemente convergente. Seja $f(x)=\lim _{n \rightarrow \infty} g_{n}(x)$. Então, para todo $\varepsilon>0$,

$$
\begin{aligned}
|f(x)-f(y)| & \leq\left|f(x)-g_{n}(x)\right|+\left|g_{n}(x)-g_{n}(y)\right|+\left|g_{n}(y)-f(y)\right| \\
& \leq 2 \varepsilon+\left|g_{n}(x)-g_{n}(y)\right|
\end{aligned}
$$


se $n$ é suficientemente grande. Fixemos $n$. A continuidade uniforme de $g_{n}$ implica que $\mid g_{n}(x)-$ $g_{n}(y) \mid<\varepsilon$ se $d(x, y)<\delta$. Portanto $|f(x)-f(y)|<3 \varepsilon$ se $d(x, y)<\delta$, provando que $f$ é contínua.

Sendo $X$ um espaço métrico compacto, denotamos por $C(X)$ o conjunto das funções contínuas $f: X \rightarrow \mathbb{R}$. O conjunto $C(X)$ é um espaço métrico completo com a métrica

$$
d(f, g)=\operatorname{máx}_{x \in X}|f(x)-g(x)|
$$

Teorema 2.2 (Ascoli-Arzela) Seja $X$ um espaço métrico compacto. Uma familia $\mathcal{H}$ de funções de $C(X)$ tem fecho compacto em $C(X)$ se, e somente se, é uniformemente limitada e eqüicontínua.

Demonstração: O Lema 2.1 mostra que se $\mathcal{H}$ é uniformemente limitada e eqüicontínua, então toda seqüência de funções $\left(f_{n}\right)_{n \geq 1}$ em $\mathcal{H}$ possui uma subseqüência que converge na métrica de $C(X)$ para um elemento de $C(X)$. Segue então que o fecho $\overline{\mathcal{H}}$ de $\mathcal{H}$ é seqüencialmente compacto e, portanto, compacto.

Reciprocamente, suponha que $\mathcal{H}$ tem fecho compacto. Temos que $\mathcal{H}$ é limitado em $C(X)$, o que é equivalente a $\mathcal{H}$ ser uma família uniformemente limitada de funções. Para provar que a família $\mathcal{H}$ é eqüicontínua, tomemos uma $\varepsilon$-cobertura de $\mathcal{H}$ por um número finito de bolas cujos centros são funções $f_{1}, f_{2}, \ldots, f_{\ell}$. Então, para toda $f \in \mathcal{H}$, existe uma $f_{j}$ com $1 \leq j \leq \ell$ tal que

$$
\left|f(x)-f_{j}(x)\right|<\varepsilon \quad \forall x \in X .
$$

Como cada uma das funções $f_{1}, f_{2}, \ldots, f_{\ell}$ é uniformemente contínua (pois $X$ é compacto), existe um $\delta>0$ tal que

$$
d(y, z)<\delta \Longrightarrow\left|f_{j}(y)-f_{j}(z)\right|<\varepsilon
$$

para todos $y, z \in X$ e para todo $1 \leq j \leq \ell$. Logo

$$
d(y, z)<\delta \Longrightarrow|f(y)-f(z)|<3 \varepsilon
$$

provando a eqüicontinuidade da família $\mathcal{H}$. 


\subsection{Operadores Compactos}

Sejam $A$ e $B$ espaços de Banach. Dizemos que o operador linear limitado $K: A \rightarrow B$ é compacto se as imagens de conjuntos limitados em $A$ têm fecho compacto em $B$. Isto é, $K$ é compacto se $\overline{K(U)}$ é compacto em $B$, em que $U=\{x \in A:\|x\| \leq 1\}$. Equivalentemente, $K: A \rightarrow B$ é compacto se, para toda seqüência limitada $\left(x_{n}\right)_{n \geq 1}$ de elementos de $A$, a seqüência $\left(K x_{n}\right)_{n \geq 1}$ possui uma subseqüência convergente.

Outra definição que nos será útil é a de operador de posto finito. Dizemos que o operador $X: A \rightarrow B$ tem posto finito se $\operatorname{dim} \operatorname{Im}(X)<+\infty$. Note que todo operador de posto finito é compacto.

Lema 2.2 Se denotarmos por $\mathcal{L}(A, B)$ o espaço dos operadores contínuos de $A$ em $B$ e denotarmos por $\mathcal{K}(A, B)$ o subespaço dos operadores compactos de $A$ em $B$, então $\mathcal{K}(A, B)$ é um subespaço fechado de $\mathcal{L}(A, B)$.

Demonstração: É fácil verificar que $\mathcal{K}(A, B)$ é um subespaço vetorial de $\mathcal{L}(A, B)$.

Seja $K_{n}: A \rightarrow B$ uma seqüência de operadores compactos que converge para o operador $K: A \rightarrow$ $B$. Mostraremos que $K$ é compacto. Suponha que $\left(x_{n}\right)_{n \geq 1}$ é uma seqüência limitada em $A$, e seja $C \in \mathbb{R}$ tal que $\left\|x_{n}\right\| \leq C$ para todo $n \geq 1$. Por compacidade, existe uma subseqüência $\left(x_{n}^{1}\right)_{n \geq 1}$ tal que $\left(K_{1} x_{n}^{1}\right)_{n \geq 1}$ é convergente em $B$. Novamente por compacidade, existe uma subseqüência $\left(x_{n}^{2}\right)_{n \geq 1}$ de $\left(x_{n}^{1}\right)_{n \geq 1}$ tal que $\left(K_{2} x_{n}^{2}\right)_{n \geq 1}$ é convergente em $B$. Recursivamente, para todo $m>1$, construímos a seqüência $\left(x_{n}^{m}\right)_{n \geq 1}$ tal que $\left(x_{n}^{m}\right)_{n \geq 1}$ é subseqüência de $\left(x_{n}^{m-1}\right)_{n \geq 1}$ e tal que $\left(K_{m} x_{n}^{m}\right)_{n \geq 1}$ é convergente em $B$.

Seja $y_{n}=x_{n}^{n}$ para todo $n \geq 1$. Então $\left(y_{n}\right)_{n \geq 1}$ é uma subseqüência de $\left(x_{n}\right)_{n \geq 1}$ tal que $\left(K_{m} y_{n}\right)_{n \geq 1}$ é convergente para todo $m$. Temos

$$
\begin{aligned}
\left\|K y_{s}-K y_{t}\right\| & \leq\left\|\left(K-K_{m}\right) y_{s}\right\|+\left\|K_{m}\left(y_{s}-y_{t}\right)\right\|+\left\|\left(K_{m}-K\right) y_{t}\right\| \\
& \leq 2 C\left\|K-K_{m}\right\|+\left\|K_{m}\left(y_{s}-y_{t}\right)\right\|,
\end{aligned}
$$

e assim $\limsup _{s, t \rightarrow \infty}\left\|K y_{s}-K y_{t}\right\| \leq 2 C\left\|K-K_{m}\right\| \rightarrow 0$ quando $m \rightarrow \infty$, o que mostra que $\left(K y_{n}\right)_{n \geq 1}$ é uma sequiência de Cauchy e portanto convergente em $B$.

Proposição 2.3 O operador limitado $K: A \rightarrow B$ é compacto se, e somente se, existe uma seqüência de operadores de posto finito $K_{n}: A \rightarrow B$ que satisfaz $\lim _{n \rightarrow \infty}\left\|K_{n}-K\right\|=0$. 
Demonstração: Suponha que $K$ é compacto. Sendo $U$ a bola unitária de $A$, temos $\overline{K(U)}$ compacto, logo $\overline{K(U)}$ contém um subconjunto denso enumerável, de onde segue que $\overline{K(A)}$ é um subespaço separável de $B$. Seja $\left\{\phi_{1}, \phi_{2}, \ldots\right\}$ uma base ortonormal de $\overline{K(A)} \subset B$ e $P_{N} y=\sum_{n=1}^{N}\left\langle y, \phi_{n}\right\rangle \phi_{n}$ a projeção ortogonal de $y$ sobre o espaço $\left[\phi_{1}, \phi_{2}, \ldots, \phi_{N}\right]$. Então $\lim _{N \rightarrow \infty}\left\|P_{N} y-y\right\|=0$ para todo $y \in K(A)$.

Seja $K_{n}=P_{n} K$. O operador $K_{n}$ tem posto finito para todo $n \geq 1$. Suponha, por absurdo, que $\limsup \left\|K-K_{n}\right\|=\varepsilon>0$. Nesse caso, existe $\left(x_{n_{k}}\right)_{k \geq 1} \in U$ tal que $\left\|\left(K-K_{n_{k}}\right) x_{n_{k}}\right\| \geq \varepsilon$ para todo $n_{k}, k \geq 1$. Como $K$ é compacto, podemos assumir que $\left(K x_{n_{k}}\right)_{k \geq 1}$ converge em $B$ (passando a uma subseqüência se necessário). Sendo $y=\lim _{k \rightarrow \infty} K x_{n_{k}}$,

$$
\begin{aligned}
\left\|\left(K-K_{n_{k}}\right) x_{n_{k}}\right\| & =\left\|\left(1-P_{n_{k}}\right) K x_{n_{k}}\right\| \\
& \leq\left\|\left(1-P_{n_{k}}\right)\left(K x_{n_{k}}-y\right)\right\|+\left\|\left(1-P_{n_{k}}\right) y\right\| \\
& \leq\left\|K x_{n_{k}}-y\right\|+\left\|\left(1-P_{n_{k}}\right) y\right\|,
\end{aligned}
$$

e esta última soma tende a zero quando $k \rightarrow \infty$. Isso contradiz o fato de que $\varepsilon>0$, logo devemos ter $\lim _{n \rightarrow \infty}\left\|K_{n}-K\right\|=0$.

Reciprocamente, se a seqüência de operadores de posto finito $K_{n}: A \rightarrow B$ é tal que $\lim _{n \rightarrow \infty} \| K_{n}-$ $K \|=0$, então $K$ é compacto, pelo Lema 2.2 e pelo fato de que todo operador de posto finito é compacto.

Lema 2.3 Sejam $A, B$ e $C$ espaços de Banach, $R \in \mathcal{L}(A, B)$ e $S \in \mathcal{L}(B, C)$. Se $R$ ou $S$ é compacto, então $S R$ é compacto.

Demonstração: Suponha que $S$ é compacto. Se $A^{\prime} \subset A$ é limitado, então $R\left(A^{\prime}\right)$ também é limitado, $\operatorname{logo} S R\left(A^{\prime}\right)$ tem fecho compacto e portanto $S R$ é compacto. Se $R$ é compacto, tome uma seqüência $\left(x_{n}\right)_{n \geq 1}$ em $A$ limitada. Então a seqüência $\left(R x_{n}\right)_{n \geq 1}$ possui uma subseqüência convergente em $B$. Logo $\left(S R x_{n}\right)_{n \geq 1}$ possui uma subseqüência convergente em $C, \operatorname{logo} S R$ é compacto.

Lema 2.4 Um operador compacto $K: A \rightarrow B$ entre espaços de Banach mapeia seqüências fracamente convergentes em seqüências convergentes (na norma). 
Demonstração: Suponha que $x_{n} \rightarrow x$. Pelo princípio da Limitação Uniforme, a seqüência $\left(\left\|x_{n}\right\|\right)_{n \geq 1}$ é limitada. Seja $y_{n}=K x_{n}$. Então $f\left(y_{n}\right)-f(y)=\left(K^{\prime} f\right)\left(x_{n}-x\right)$ para todo $f \in B^{\prime}$, e portanto $y_{n}$ converge fracamente para $y=K x$ em $B$. Suponha que $y_{n}$ não seja convergente a $y$ na norma. Então existem um $\varepsilon>0$ e uma subseqüência $\left(y_{n_{k}}\right)_{k \geq 1}$ de $\left(y_{n}\right)_{n \geq 1}$ tais que $\left\|y_{n_{k}}-y\right\| \geq \varepsilon$. Como a seqüência $\left(x_{n_{k}}\right)_{k \geq 1}$ é limitada e $K$ é compacto, a seqüência $\left(y_{n_{k}}\right)_{k \geq 1}$ possui uma subseqüência que converge para um $\tilde{y} \neq y$. Esta subseqüência deve então convergir fracamente para $\tilde{y}$, um absurdo porque $y_{n}$ converge fracamente para $y$. Logo $y_{n} \rightarrow y$.

\subsection{Operadores de Fredholm}

Sejam $A$ e $B$ espaços de Hilbert. Um operador $X: A \rightarrow B$ é chamado um operador de Fredholm se

1. $\operatorname{dim}(\operatorname{Ker} X)<+\infty$;

2. $\operatorname{Im} X$ é subespaço fechado e $\operatorname{codim}(\operatorname{Im} X):=\operatorname{dim}\left(\frac{B}{\operatorname{Im} X}\right)<+\infty$.

Se $X: A \rightarrow B$ é um operador de Fredholm, definimos seu índice $i_{F}(X)$ por

$$
i_{F}(X)=\operatorname{dim}(\operatorname{Ker} X)-\operatorname{codim}(\operatorname{Im} X) .
$$

Lema 2.5 Sejam $H$ um espaço de Hilbert, $M \subset H$ um subespaço fechado, e $V \subset H$ um subespaço de dimensão finita. Então $M+V$ é um subespaço fechado. Em particular, se $\operatorname{codim}(M)<+\infty e$ $W \subset H$ é um subespaço tal que $M \subset W$, então $W$ é fechado e $\operatorname{codim}(W)<+\infty$.

Demonstração: Seja $P: H \rightarrow M$ a projeção ortogonal e seja $V_{0}=(I-P) V$. O espaço $V_{0}$ é fechado, pois $\operatorname{dim}\left(V_{0}\right) \leq \operatorname{dim}(V)<+\infty$. Também é claro que $M+V=M \stackrel{\perp}{\oplus} V_{0}$. Disso segue que $M+V$ é fechado, pois a seqüência $z_{n}=m_{n}+v_{n}$ em $M+V$ é convergente se $m_{n}$ é uma seqüência convergente em $M$ e $v_{n}$ é uma seqüência convergente em $V_{0}$.

Se $\operatorname{codim}(M)<+\infty$ e $M \subset W$, existe um subespaço de dimensão finita $V \subset H$ tal que $M+V=$ $W, \operatorname{logo} W$ é subespaço fechado e $\operatorname{codim}(W) \leq \operatorname{codim}(M)<+\infty$. 
Lema 2.6 Se $K: A \rightarrow B$ é um operador de posto finito entre os espaços de Hilbert $A$ e $B$, então existem seqüências $\left(\phi_{n}\right)_{n=1}^{k} \subset A e\left(\psi_{n}\right)_{n=1}^{k} \subset B$ tais que

1. $K x=\sum_{n=1}^{k}\left\langle x, \phi_{n}\right\rangle \psi_{n}$ para todo $x \in A$;

2. $K^{*} y=\sum_{n=1}^{k}\left\langle y, \psi_{n}\right\rangle \phi_{n}$ para todo $y \in B$. Em particular, $K^{*}$ tem posto finito;

Assumindo que $A=B$, valem também

3. $\operatorname{dim} \operatorname{Ker}(I+K)<+\infty$;

4. $\operatorname{dim} \operatorname{Coker}(I+K):=\operatorname{codim} \operatorname{Im}(I+K)<+\infty, \operatorname{Im}(I+K)$ é fechada e $\operatorname{Im}(I+K)=\operatorname{Ker}\left(I+K^{*}\right)^{\perp}$.

\section{Demonstração:}

1. Basta tomar $\left\{\psi_{1}, \ldots, \psi_{k}\right\}$ base ortonormal de $\operatorname{Im}(K)$. Então, se $x \in A$,

$$
K x=\sum_{n=1}^{k}\left\langle K x, \psi_{n}\right\rangle \psi_{n}=\sum_{n=1}^{k}\left\langle x, K^{*} \psi_{n}\right\rangle \psi_{n}
$$

e tomamos $\phi_{n}=K^{*} \psi_{n}$.

2. Dado $y \in B$, para todo $x \in A$ temos

$$
\begin{aligned}
\langle K x, y\rangle & =\left\langle\sum_{n=1}^{k}\left\langle x, \phi_{n}\right\rangle \psi_{n}, y\right\rangle \\
& =\sum_{n=1}^{k}\left\langle\left\langle x, \phi_{n}\right\rangle \psi_{n}, y\right\rangle \\
& =\sum_{n=1}^{k}\left\langle x, \phi_{n}\right\rangle\left\langle y, \psi_{n}\right\rangle=\left\langle x, K^{*} y\right\rangle,
\end{aligned}
$$

de onde concluímos que $K^{*} y=\sum_{n=1}^{k}\left\langle y, \psi_{n}\right\rangle \phi_{n}$.

3. $\operatorname{Ker}(I+K)=\{x \in A: x=K(-x)\} \subset \operatorname{Im}(K)$, logo tem dimensão finita. 
4. Se $x \in \operatorname{Ker}(K)$, então $x=(I+K) x \in \operatorname{Im}(I+K)$, logo $\operatorname{Ker}(K) \subset \operatorname{Im}(I+K)$. Do item 1 temos $\left\{\phi_{1}, \ldots, \phi_{k}\right\}^{\perp} \subset \operatorname{Ker}(K)$, o que implica $A=\operatorname{Ker}(K)+\left[\phi_{1}, \ldots, \phi_{k}\right]$, onde $\left[\phi_{1}, \ldots, \phi_{k}\right]$ representa o espaço gerado por $\left\{\phi_{1}, \ldots, \phi_{k}\right\}$. Portanto codim $\operatorname{Ker}(K)<+\infty$. Pelo Lema 2.5, $\operatorname{Im}(I+K)$ é fechada e codim $\operatorname{Im}(I+K)<+\infty$. Finalmente temos $\operatorname{Im}(I+K)=\operatorname{Ker}\left(I+K^{*}\right)^{\perp}$, pois se $X: A \rightarrow B$ é um operador limitado, então $\overline{\operatorname{Im}(X)}=\operatorname{Ker}\left(X^{*}\right)^{\perp}$. Podemos ver isso da seguinte forma: $y \in \operatorname{Ker}\left(X^{*}\right) \Longleftrightarrow 0=\left\langle X^{*} y, x\right\rangle=\langle y, X x\rangle$ para todo $x \in A$, o que equivale a dizer que $y \in \operatorname{Im}(X)^{\perp}$. Temos então $\overline{\operatorname{Im}(X)}=\operatorname{Im}(X)^{\perp \perp}=\operatorname{Ker}\left(X^{*}\right)^{\perp}$.

Estes dois lemas nos permitem demonstrar a proposição abaixo, que nos fornece outra caracterização dos operadores de Fredholm.

Proposição 2.4 O operador linear limitado $X: A \rightarrow B$ é Fredholm se, e somente se, existe um operador limitado $Y: B \rightarrow A$ tal que $X Y-I$ e $Y X-I$ são ambos compactos (de fato, podemos escolher $Y$ de modo que $X Y-I$ e $Y X-I$ têm posto finito).

Demonstração: Suponha que $X$ é Fredholm. A restrição $\tilde{X}: \operatorname{Ker}(X)^{\perp} \rightarrow \operatorname{Im} X$ é uma bijeção linear limitada entre espaços de Hilbert ( $\operatorname{Im} X$ é fechado em $B$ e portanto é um espaço de Hilbert). Pelo teorema da Aplicação Aberta, a aplicação $\tilde{X}^{-1}$ é limitada. Seja $P: B \rightarrow \operatorname{Im} X$ a projeção ortogonal e defina $Y=\tilde{X}^{-1} P$. Então $Y X-I=\tilde{X}^{-1} P X-I=\tilde{X}^{-1} X-I=-Q$, em que $Q$ é a projeção ortogonal sobre $\operatorname{Ker} X$. Analogamente, $X Y-I=X \tilde{X}^{-1} P-I=-(I-P)$. Mas $Q$ e $I-P$ são projeções de posto finito, logo compactas, e portanto $X Y-I$ e $Y X-I$ são operadores compactos.

Para demonstrar a recíproca, primeiro mostramos que o operador $Y: B \rightarrow A$ pode ser modificado de modo que $X Y-I$ e $Y X-I$ tenham posto finito. Para tanto, seja $G=Y X-I$ ( $G$ é compacto) e escolha uma aproximação $G_{1}$ de $G$ que tenha posto finito e tal que $G=G_{1}+E$, com $\|E\|<1$. Defina $Y_{L}: B \rightarrow A$ por $Y_{L}=(I+E)^{-1} Y$. Como $Y X=(I+E)+G_{1}$,

$$
Y_{L} X=(I+E)^{-1} Y X=I+(I+E)^{-1} G_{1}=I+K_{L},
$$

em que $K_{L}$ tem posto finito. Analogamente, existe um operador limitado $Y_{R}: B \rightarrow A$ e um operador de posto finito $K_{R}$ tal que $X Y_{R}=I+K_{R}$. Note que $Y_{L} X Y_{R}=Y_{R}+K_{L} Y_{R}=Y_{L}+Y_{L} K_{R} \Rightarrow$ $Y_{L}-Y_{R}=K_{L} Y_{R}-Y_{L} K_{R}=S$, e $S$ tem posto finito. Portanto $X Y_{L}=X\left(Y_{R}+S\right)=I+K_{R}+X S$, 
logo $X Y_{L}-I=K_{R}+X S$ tem posto finito. Assim, $Y_{L}$ é tal que $X Y_{L}-I$ e $Y_{L} X-I$ têm ambos posto finito.

Agora, portanto, assumimos que $X Y-I=G_{1}$ e $Y X-I=G_{2}$ têm posto finito. Temos $\operatorname{Ker}(X) \subset \operatorname{Ker}(Y X)=\operatorname{Ker}\left(I+G_{1}\right)$ e $\operatorname{Im}(X) \supset \operatorname{Im}(X Y)=\operatorname{Im}\left(I+G_{2}\right)$. O resultado segue dos Lemas 2.5 e 2.6 .

Lema 2.7 Se $A$ e $B$ são espaços de Hilbert, o operador limitado $F: A \rightarrow B$ é Fredholm se, e somente se, existem decomposições ortogonais $A=A_{1} \oplus A_{2}$ e $B=B_{1} \oplus B_{2}$ tais que

1. $A_{1}$ e $B_{1}$ são subespaços fechados;

2. $A_{2}$ e $B_{2}$ tem dimensão finita;

3. F pode ser escrito na forma

$$
F=\left(\begin{array}{cc}
F_{11} & F_{12} \\
F_{21} & F_{22}
\end{array}\right): \begin{array}{ccc}
A_{1} & & B_{1} \\
A_{2} & & \oplus \\
B_{2}
\end{array}
$$

com $F_{11}: A_{1} \rightarrow B_{1}$ limitado e inversivel.

Além disso, dada essa decomposição, $i_{F}(F)=\operatorname{dim}\left(A_{2}\right)-\operatorname{dim}\left(B_{2}\right)$.

Demonstração: Se $F$ é Fredholm, tomamos $A_{1}=\operatorname{Ker}(F)^{\perp}, A_{2}=\operatorname{Ker}(F), B_{1}=\operatorname{Im}(F), B_{2}=$ $\operatorname{Im}(F)^{\perp}$. Dessa forma temos $F=\left(\begin{array}{cc}F_{11} & 0 \\ 0 & 0\end{array}\right)$, em que $F_{11}=\left.F\right|_{A_{1}}$ é inversível.

Para demonstrar a recíproca, seja $F$ dado pela equação $(2.2)$, e considere $G=\left(\begin{array}{cc}F_{11}^{-1} & 0 \\ 0 & 0\end{array}\right)$. Temos

$$
G F=\left(\begin{array}{cc}
I & F_{11}^{-1} F_{12} \\
0 & 0
\end{array}\right)=\left(\begin{array}{cc}
I & 0 \\
0 & I
\end{array}\right)+\left(\begin{array}{cc}
0 & F_{11}^{-1} F_{12} \\
0 & -I
\end{array}\right)
$$

logo $G F-I$ tem posto finito. Analogamente, $F G-I$ tem posto finito, e portanto $F$ é Fredholm. 
Para calcularmos $i_{F}(F)$, perceba que $\left(\begin{array}{l}x_{1} \\ x_{2}\end{array}\right) \in \operatorname{Ker}(F)$ se, e somente se,

$$
\begin{aligned}
& F_{11} x_{1}+F_{12} x_{2}=0 \\
& F_{21} x_{1}+F_{22} x_{2}=0
\end{aligned}
$$

o que ocorre se, e somente se, $x_{1}=-F_{11}^{-1} F_{12} x_{2}$ e $\left(-F_{21} F_{11}^{-1} F_{12}+F_{22}\right) x_{2}=0$. Se $D=\left(-F_{21} F_{11}^{-1} F_{12}+\right.$ $\left.F_{22}\right): A_{2} \rightarrow B_{2}$, então a função

$$
x_{2} \in \operatorname{Ker}(D) \mapsto\left(\begin{array}{c}
-F_{11}^{-1} F_{12} x_{2} \\
x_{2}
\end{array}\right) \in \operatorname{Ker}(F)
$$

é um isomorfismo linear de espaços vetoriais e portanto $\operatorname{Ker}(F) \cong \operatorname{Ker}(D)$. Como

$$
F^{*}=\left(\begin{array}{cc}
F_{11}^{*} & F_{21}^{*} \\
F_{12}^{*} & F_{22}^{*}
\end{array}\right): \begin{array}{ccc}
B_{1} & & A_{1} \\
B_{2} & \longrightarrow & \oplus \\
A_{2}
\end{array}
$$

um raciocínio análogo mostra que $\operatorname{Ker}\left(F^{*}\right) \cong \operatorname{Ker}\left(D^{*}\right)$. Logo

$$
\begin{aligned}
i_{F}(F) & =\operatorname{dim}(\operatorname{Ker}(F))-\operatorname{codim}(\operatorname{Im}(F)) \\
& =\operatorname{dim}(\operatorname{Ker}(D))-\operatorname{dim}(\operatorname{Coker}(F)) \\
& =\operatorname{dim}(\operatorname{Ker}(D))-\operatorname{dim}\left(\operatorname{Ker}\left(F^{*}\right)\right) \\
& =\operatorname{dim}(\operatorname{Ker}(D))-\operatorname{dim}\left(\operatorname{Ker}\left(D^{*}\right)\right)=i_{F}(D) .
\end{aligned}
$$

Na equação acima, usamos o fato que $B=\operatorname{Im}(F) \oplus \operatorname{Im}(F)^{\perp}=\operatorname{Im}(F) \oplus \operatorname{Ker}\left(F^{*}\right)$, o que implica $\operatorname{Coker}(F)=B / \operatorname{Im}(F) \cong \operatorname{Ker}\left(F^{*}\right)$. Finalmente, pelo teorema do Núcleo e da Imagem, temos

$$
\begin{aligned}
\operatorname{dim}\left(A_{2}\right) & =\operatorname{dim}(\operatorname{Ker}(D))+\operatorname{dim}(\operatorname{Im}(D)) \\
& =\operatorname{dim}(\operatorname{Ker}(D))+\left(\operatorname{dim}\left(B_{2}\right)-\operatorname{dim}\left(B_{2} / \operatorname{Im}(D)\right)\right.
\end{aligned}
$$

de onde segue $i_{F}(D)=\operatorname{dim}\left(A_{2}\right)-\operatorname{dim}\left(B_{2}\right)$. 
Proposição 2.5 Sejam $A$ e $B$ espaços de Hilbert, $F: A \rightarrow B$ um operador de Fredholm $e K: A \rightarrow B$ um operador compacto. Então $F+K$ é um operador de Fredholm e $i_{F}(F+K)=i_{F}(F)$.

Demonstração: Sabemos que $F$ pode ser escrito na forma dada pela equação $(2.2)$, com $F_{11}: A_{1} \rightarrow$ $B_{1}$ inversível. Seja $E: A \rightarrow B$ um operador limitado cuja decomposição de acordo com a equação (2.2) é

$$
E=\left(\begin{array}{ll}
E_{11} & E_{12} \\
E_{21} & E_{22}
\end{array}\right)
$$

e escolhamos $E$ de modo que $\|E\|$ seja suficientemente pequeno, de modo a garantir que $\left\|E_{11}\right\|$ também é pequeno o suficiente para que $F_{11}+E_{11}$ ainda seja inversível (isso é possível pois os operadores inversíveis formam um conjunto aberto). $\operatorname{Logo} F+E=\left(\begin{array}{cc}F_{11}+E_{11} & * \\ * & *\end{array}\right)$ e, pelo Lema $2.7, F+E$ é um operador de Fredholm e $i_{F}(F+E)=\operatorname{dim}\left(A_{2}\right)-\operatorname{dim}\left(B_{2}\right)=i_{F}(F)$. Isto é, mostramos que os operadores de Fredholm formam um conjunto aberto dos operadores limitados, e que se $E: A \rightarrow B$ tem norma suficientemente pequena, então $i_{F}(F+E)=i_{F}(F)$.

Como $F$ é um operador de Fredholm, a Proposição 2.4 garante a existência de um operador $G: B \rightarrow A$ tal que $G_{1}=G F-I$ e $G_{2}=F G-I$ são ambos compactos. Mas então $G(F+K)-I=$ $G_{1}+G K$ e $(F+K) G-I=G_{2}+K G$ são compactos, logo $F+K$ é um operador de Fredholm pela Proposição 2.4. Consideremos a função de variável real $f(t)=i_{F}(F+t K)$. Mostramos no parágrafo anterior que $f$ é uma função localmente constante para cada $t \in \mathbb{R}$, logo é constante. Em particular, $i_{F}(F+K)=f(1)=f(0)=i_{F}(F)$.

\subsection{Teorema Espectral}

Seja $H$ um espaço de Hilbert sobre $\mathbb{R}$ ou $\mathbb{C}$.

Proposição 2.6 Sejae $T: H \rightarrow H$ um operador limitado auto-adjunto. Então

$$
\|T\|=\sup _{x \neq 0} \frac{|\langle x, T x\rangle|}{\|x\|^{2}}
$$


Além disso, se existir um elemento não nulo $v \in H$ tal que

$$
\|T\|=\frac{|\langle v, T v\rangle|}{\|v\|^{2}}
$$

então $v$ é um autovetor de $T$ com $T v=\lambda v$, em que $\lambda= \pm\|T\|$.

Demonstração: Seja $M=\sup _{x \neq 0} \frac{|\langle x, T x\rangle|}{\|x\|^{2}}$. Como $|\langle x, T x\rangle| \leq\|x\|\|T x\| \leq\|T\|\|x\|^{2}$, temos $M \leq$ $\|T\|$. Reciprocamente, sejam $u, v \in H$ :

$$
\begin{aligned}
\langle u+v, T(u+v)\rangle-\langle u-v, T(u-v)\rangle & =2(\langle u, T v\rangle+\langle v, T u\rangle) \\
& =4 \Re\langle u, T v\rangle .
\end{aligned}
$$

Portanto, se $\|u\|=\|v\|=1$, segue que

$$
|\Re\langle u, T v\rangle| \leq \frac{M}{4}\left[\|u+v\|^{2}+\|u-v\|^{2}\right]=\frac{M}{4}\left[2\|u\|^{2}+2\|v\|^{2}\right]=M .
$$

Substituindo $u$ por $e^{i \theta} u$, em que $\theta$ é escolhido de modo que $e^{i \theta}\langle u, T v\rangle \in \mathbb{R}$, temos $|\langle u, T v\rangle| \leq M$ para todos $u, v$ de norma 1. Agora, se $v \neq 0$ é tal que $\|T\|=|\langle v, T v\rangle| /\|v\|^{2}$, pela desigualdade de Cauchy-Schwarz temos

$$
\|T\|=\frac{|\langle v, T v\rangle|}{\|v\|^{2}} \leq \frac{\|T v\|}{\|v\|} \leq\|T\|
$$

o que implica $|\langle v, T v\rangle|=\|T v\|\|v\|$. Mas, na desigualdade de Cauchy-Schwarz, a igualdade só pode ocorrer se $v$ e $T v$ forem linearmente dependentes, isto é, existe $\lambda \in \mathbb{C}$ tal que $T v=\lambda v$. Substituindo na equação (2.3), obtemos $|\lambda|=\|T\|$, e como os autovalores de um operador auto-adjunto são reais, temos $\lambda= \pm\|T\|$.

Proposição 2.7 Se $T \in \mathcal{L}(H)$ um operador compacto auto-adjunto, então $\|T\|$ ou - $\|T\|$ é um autovalor de $T$.

Demonstração: Se $T=0$ o resultado é trivial, portanto assumimos $T \neq 0$. Pela Proposição 2.6 existe uma seqüência $\left(v_{n}\right)_{n \geq 1}$ de elementos de $H$ tal que $\left\|v_{n}\right\|=1$ para todo $n \geq 1$ e

$$
\frac{\left|\left\langle v_{n}, T v_{n}\right\rangle\right|}{\left\|v_{n}\right\|^{2}}=\left|\left\langle v_{n}, T v_{n}\right\rangle\right| \rightarrow\|T\| \quad \text { quando } \quad n \rightarrow \infty .
$$


Passando a uma subseqüência se necessário, podemos assumir que existe $\lambda=\lim _{n \rightarrow \infty}\left\langle v_{n}, T v_{n}\right\rangle$ e $\lambda=$ $\pm\|T\|$. Passando novamente a uma subseqüência se necessário, podemos supor que $T v_{n}$ também converge (pois $T$ é compacto). Nessas condições,

$$
\begin{aligned}
0 & \leq\left\|T v_{n}-\lambda v_{n}\right\|^{2}=\left\|T v_{n}\right\|^{2}-2 \lambda\left\langle T v_{n}, v_{n}\right\rangle+\lambda^{2} \\
& \leq \lambda^{2}-2 \lambda\left\langle T v_{n}, v_{n}\right\rangle+\lambda^{2} \rightarrow \lambda^{2}-2 \lambda^{2}+\lambda^{2}=0 \text { quando } n \rightarrow \infty
\end{aligned}
$$

Assim, quando $n \rightarrow \infty$,

$$
\left(T v_{n}-\lambda v_{n}\right) \rightarrow 0
$$

e $v=\lim _{n \rightarrow \infty} v_{n}=\frac{1}{\lambda} \lim _{n \rightarrow \infty} T v_{n}$ existe. Pela continuidade do produto interno, $\|v\|=1 \neq 0$. Pela equação (2.4), obtemos $T v=\lambda v$.

Lema 2.8 Se $T: H \rightarrow H$ é um operador auto-adjunto e $M$ é um subespaço invariante por $T$, então $M^{\perp}$ também é invariante por $T$.

Demonstração: Seja $x \in M$ e $y \in M^{\perp}$. Então $T x \in M$ e portanto

$$
0=\langle T x, y\rangle=\langle x, T y\rangle \quad \text { para todo } x \in M,
$$

$\operatorname{logo} T y \in M^{\perp}$.

Teorema 2.3 (Teorema Espectral) Se $T: H \rightarrow H$ é um operador compacto e auto-adjunto, então

1. Existe pelo menos um autovalor $\lambda= \pm\|T\|$;

2. T possui no máximo uma quantidade enumerável de autovalores não nulos, $\left(\lambda_{n}\right)_{n=1}^{N}$, em que podemos ter $N=\infty$ (a menos que $T$ tenha posto finito, teremos $N=\infty$ );

3. Os $\lambda_{n}$ 's podem ser dispostos em seqüência de modo que $\left|\lambda_{n}\right| \geq\left|\lambda_{n+1}\right|$ para todo $n$. Se $N=\infty$, então $\lim _{n \rightarrow \infty}\left|\lambda_{n}\right|=0$. Em particular, todo autoespaço de $T$ correspondente a um autovalor não nulo tem dimensão finita; 
4. Os autovetores $\left(\phi_{n}\right)_{n=1}^{N}$ podem ser escolhidos de modo a constituirem um conjunto ortonormal tal que $H=\overline{\left[\phi_{1}, \phi_{2}, \ldots\right]} \oplus \operatorname{Ker}(T)$;

5. Usando os autovetores $\left(\phi_{n}\right)_{n=1}^{N}$ acima, temos

$$
T \psi=\sum_{n=1}^{N} \lambda_{n}\left\langle\psi, \phi_{n}\right\rangle \phi_{n} \quad \text { para todo } \psi \in H
$$

6. O espectro de $T$ é $\sigma(T)=\{0\} \cup \bigcup_{n=1}^{\infty}\left\{\lambda_{n}\right\}$.

Demonstração: Os autovalores e autovetores serão determinados recursivamente. Seja $\lambda_{1}= \pm\|T\|$ dado pela Proposição 2.7 e $\phi_{1} \in H$ tal que $\left\|\phi_{1}\right\|=1$ e $T \phi_{1}=\lambda_{1} \phi_{1}$. Seja $M_{1}=\left[\phi_{1}\right]$, de modo que $T\left(M_{1}\right) \subset M_{1}$. Pelo Lema 2.8, $T\left(M_{1}^{\perp}\right) \subset M_{1}^{\perp}$. Defina $T_{1}: M_{1}^{\perp} \rightarrow M_{1}^{\perp}$ por $T_{1}=\left.T\right|_{M_{1}^{\perp}}$. Então $T_{1}$ é um operador compacto. Se $T_{1}=0$, nada mais resta a fazer.

Se $T_{1} \neq 0$, pela Proposição 2.7 existem $\lambda_{2}= \pm\left\|T_{1}\right\|$ e $\phi_{2} \in M_{1}^{\perp}$ tais que $\left\|\phi_{2}\right\|=1$ e $T_{1} \phi_{2}=\lambda_{2} \phi_{2}$. Seja $M_{2}=\left[\phi_{1}, \phi_{2}\right]$. Temos novamente que $T\left(M_{2}\right) \subset M_{2}$ e portanto o operador $T_{2}: M_{2}^{\perp} \rightarrow M_{2}^{\perp}$ dado por $T_{2}=\left.T\right|_{M_{2}^{\perp}}$ é compacto. Se $T_{2}=0$, nada resta a fazer.

Se $T_{2} \neq 0$, repetimos o procedimento acima. Continuamos desta forma indefinidamente ou até encontrarmos $T_{N}=0$, e construímos assim a seqüência $\left(\lambda_{n}\right)_{n=1}^{N}$ com a propriedade que $\left|\lambda_{n}\right| \geq\left|\lambda_{n+1}\right|$, e também a seqüência ortonormal $\left(\phi_{n}\right)_{n=1}^{N}$. Temos ainda

$$
\left|\lambda_{i}\right|=\sup _{\phi \perp\left\{\phi_{1}, \phi_{2}, \ldots, \phi_{i-1}\right\}} \frac{\|T \phi\|}{\|\phi\|} .
$$

Mostremos agora que $\lim _{n \rightarrow \infty}\left|\lambda_{n}\right|=0$ quando $N=\infty$. Se $\lim _{n \rightarrow \infty}\left|\lambda_{n}\right|>0$, então existe $\varepsilon>0$ tal que $\left|\lambda_{i}\right| \geq \varepsilon$ para todo $i$, e nesse caso a seqüência $\left(\phi_{i} / \lambda_{i}\right)_{n \geq 1}$ em $H$ é limitada por $\varepsilon^{-1}$. Pela compacidade de $T$, existe uma subseqüência $\left(\phi_{i_{k}} / \lambda_{i_{k}}\right)$ tal que $T \phi_{i_{k}} / \lambda_{i_{k}}=\phi_{i_{k}}$ é convergente, o que é impossível já que $\left\{\phi_{1}, \phi_{2}, \ldots\right\}$ é ortonormal. Logo $\lim _{n \rightarrow \infty}\left|\lambda_{n}\right|=0$.

Seja $M=\left[\phi_{1}, \phi_{2}, \ldots, \phi_{N}\right]$ se $N<\infty$ ou $M=\left[\phi_{1}, \phi_{2}, \ldots\right]$ se $N=\infty$. Então $T(M) \subset M$ e portanto $T\left(M^{\perp}\right) \subset M^{\perp}$. Usando a equação (2.5), se $N=\infty$ obtemos

$$
\left\|T_{M^{\perp}}\right\| \leq\left\|T_{M_{i}^{\perp}}\right\|=\left|\lambda_{i}\right| \rightarrow 0 \quad \text { quando } i \rightarrow \infty
$$


mostrando que $T_{M^{\perp}}=0$. Quando $N<\infty$, é claro que $T_{M^{\perp}}=0$. Seja $P_{0}$ a projeção ortogonal sobre $M^{\perp}$. Para todo $\psi \in H$,

$$
\psi=P_{0} \psi+\left(1-P_{0}\right) \psi=P_{0} \psi+\sum_{i=1}^{N}\left\langle\psi, \phi_{i}\right\rangle \phi_{i}
$$

e

$$
T \psi=T P_{0} \psi+T \sum_{i=1}^{N}\left\langle\psi, \phi_{i}\right\rangle \phi_{i}=\sum_{i=1}^{N} \lambda_{i}\left\langle\psi, \phi_{i}\right\rangle \phi_{i} .
$$

Como $\left\{\lambda_{1}, \lambda_{2}, \ldots\right\} \subset \sigma(T)$ e $\sigma(T)$ é fechado, segue que $0 \in \sigma(T), \operatorname{logo}\{0\} \cup \bigcup_{n=1}^{\infty}\left\{\lambda_{n}\right\} \subset \sigma(T)$. Seja agora $z \notin\{0\} \cup \bigcup_{n=1}^{\infty}\left\{\lambda_{n}\right\}$ e seja $d$ a distância de $z$ ao conjunto $\{0\} \cup \bigcup_{n=1}^{\infty}\left\{\lambda_{n}\right\}$. Temos $d>0$, pois $\lim _{n \rightarrow \infty} \lambda_{n}=0$. Além disso,

$$
(T-z I) \psi=\sum_{i=1}^{N}\left\langle\psi, \phi_{i}\right\rangle\left(\lambda_{i}-z\right) \phi_{i}-z P_{0} \psi,
$$

$(T-z I)^{-1}$ existe,

$$
(T-z I)^{-1} \psi=\sum_{i=1}^{N}\left\langle\psi, \phi_{i}\right\rangle\left(\lambda_{i}-z\right)^{-1} \phi_{i}-z^{-1} P_{0} \psi
$$

e

$$
\begin{aligned}
\left\|(T-z I)^{-1} \psi\right\|^{2} & =\sum_{i=1}^{N}\left|\left\langle\psi, \phi_{i}\right\rangle\right|^{2} \frac{1}{\left|\lambda_{i}-z\right|^{2}}+\frac{1}{|z|^{2}}\left\|P_{0} \psi\right\|^{2} \\
& \leq \frac{1}{d^{2}}\left(\sum_{i=1}^{N}\left|\left\langle\psi, \phi_{i}\right\rangle\right|^{2}+\left\|P_{0} \psi\right\|^{2}\right)=\frac{\|\psi\|^{2}}{d^{2}} .
\end{aligned}
$$

Mostramos então que $(T-z I)^{-1}$ existe e $\left\|(T-z I)^{-1}\right\| \leq d^{-1}<+\infty$, logo $(T-z I)^{-1}$ é limitada e concluímos que $z \notin \sigma(T)$, encerrando a demonstração. 


\section{Capítulo 3}

\section{Os espaços $L^{p}$ e os espaços de Sobolev}

Neste capítulo recordamos alguns resultados fundamentais a respeito dos espaços $L^{p}$ e dos espaços de Sobolev.

\subsection{Os espaços $L^{p}$}

Definição 3.1 Seja $p \in \mathbb{R}$ com $1 \leq p<\infty$ e $\Omega$ um aberto de $\mathbb{R}^{n}$. Definimos

$$
L^{p}(\Omega)=\left\{u: \Omega \rightarrow \mathbb{R}: \text { u é mensurável e } \int_{\Omega}|u|^{p}<\infty\right\} .
$$

A norma em $L^{p}(\Omega)$ é definida por

$$
\|u\|_{L^{p}}=\left(\int_{\Omega}|u|^{p}\right)^{1 / p} .
$$

\section{Definição 3.2 Definimos}

$$
\begin{gathered}
L^{\infty}(\Omega)=\{u: \Omega \rightarrow \mathbb{R}: \text { u é mensurável } e \exists C \in \mathbb{R} \text { tal que }|u(x)| \leq C \\
\text { quase sempre em } \Omega\} .
\end{gathered}
$$

A norma em $L^{\infty}(\Omega)$ é definida por

$$
\|u\|_{L^{\infty}}=\inf \{C \in \mathbb{R}:|u(x)| \leq C \text { quase sempre em } \Omega\} .
$$


Nos espaços $L^{p}$, duas desigualdades importantes são as de Hölder e Minkowski. De fato, a desigualdade de Minkowski é conseqüência da desigualdade de Hölder e é usada para mostrar que de fato $\|\cdot\|_{L^{p}}$ como definimos constitui uma norma no espaço $L^{p}(\Omega), 1 \leq p \leq \infty$.

Teorema 3.1 (Desigualdade de Hölder) Seja $1<p<\infty$ e seja $q$ tal que $\frac{1}{p}+\frac{1}{q}=1$. Sejam $u \in L^{p}(\Omega)$ e $v \in L^{q}(\Omega)$. Então $u v \in L^{1}(\Omega) e$

$$
\int_{\Omega}|u v| \leq\left(\int_{\Omega}|u|^{p}\right)^{1 / p}\left(\int_{\Omega}|v|^{q}\right)^{1 / q} .
$$

Observação: Se $p=q=2$, a desigualdade de Hölder é conhecida como desigualdade de CauchySchwarz.

Corolário 3.1 Se $1 \leq p \leq q \leq r<\infty$ e $u \in L^{r}(\Omega)$, então

$$
\|u\|_{L^{q}} \leq\|u\|_{L^{p}}^{\lambda}\|u\|_{L^{r}}^{1-\lambda}
$$

em que $\frac{1}{q}=\frac{\lambda}{p}+\frac{(1-\lambda)}{r}$.

Demonstração: Seja $\alpha=\lambda q, \beta=(1-\lambda) q$. Sendo $z=\frac{p}{\lambda q}$ e $y=\frac{r}{(1-\lambda) q}$, temos

$$
\frac{1}{z}+\frac{1}{y}=\frac{\lambda q}{p}+\frac{(1-\lambda) q}{r}=\frac{\lambda q}{p}+\left(1-\frac{\lambda q}{p}\right)=1 .
$$

Portanto, pela desigualdade de Hölder,

$$
\int_{\Omega}|u|^{q}=\int_{\Omega}|u|^{\alpha}|u|^{\beta} \leq\left(\int_{\Omega}|u|^{\alpha z}\right)^{1 / z}\left(\int_{\Omega}|u|^{\beta y}\right)^{1 / y},
$$

que é equivalente a

$$
\left(\int_{\Omega}|u|^{q}\right)^{1 / q} \leq\left(\int_{\Omega}|u|^{p}\right)^{\lambda / p}\left(\int_{\Omega}|u|^{r}\right)^{(1-\lambda) / r} .
$$

A desigualdade de Hölder pode ser generalizada: 
Corolário 3.2 Seja $m \geq 2$ um inteiro. Se $1<p_{1}, p_{2}, \ldots, p_{m}<\infty$ são tais que $\sum_{i=1}^{m} \frac{1}{p_{i}}=1$, e se $u_{i} \in L^{p_{i}}(\Omega)$ para $1 \leq i \leq m$, então

$$
\int_{\Omega}\left|u_{1} u_{2} \cdots u_{m}\right| \leq \prod_{i=1}^{m}\left(\int_{\Omega}\left|u_{i}\right|^{p_{i}}\right)^{1 / p_{i}}
$$

Demonstração: Procedemos por indução em $m$. O caso $m=2$ é a desigualdade de Hölder. Se $\sum_{i=1}^{m} \frac{1}{p_{i}}=1$, seja $r$ tal que $\frac{1}{r}+\frac{1}{p_{m}}=1$, isto é, $\frac{1}{r}=\sum_{i=1}^{m-1} \frac{1}{p_{i}}$. Observando que $\sum_{i=1}^{m-1} \frac{1}{p_{i} / r}=1$, temos

$$
\begin{aligned}
\int_{\Omega}\left|u_{1} \cdots u_{m}\right| & \leq\left(\int_{\Omega}\left|u_{1} \cdots u_{m-1}\right|^{r}\right)^{1 / r}\left(\int_{\Omega}\left|u_{m}\right|^{p_{m}}\right)^{1 / p_{m}} \\
& \leq\left(\int_{\Omega}\left|u_{m}\right|^{p_{m}}\right)^{1 / p_{m}}\left(\prod_{i=1}^{m-1}\left(\int_{\Omega}\left|u_{i}^{r}\right|^{p_{i} / r}\right)^{r / p_{i}}\right)^{1 / r} \\
& =\prod_{i=1}^{m}\left(\int_{\Omega}\left|u_{i}\right|^{p_{i}}\right)^{1 / p_{i}} .
\end{aligned}
$$

Teorema 3.2 (Desigualdade de Minkowski) Seja $1<p<\infty$, e sejam $u, v \in L^{p}(\Omega)$. Então

$$
\left(\int_{\Omega}(|u|+|v|)^{p}\right)^{1 / p} \leq\left(\int_{\Omega}|u|^{p}\right)^{1 / p}+\left(\int_{\Omega}|v|^{p}\right)^{1 / p}
$$

Recordamos ainda que os espaços $L^{p}(\Omega)$, quando vistos como espaços métricos, não são espaços de funções, e sim espaços de classes de equivalências de funções. Isso ocorre porque $\|u\|_{L^{p}}=0$ para toda $u$ que é zero exceto num conjunto de medida nula. Assim, as classes de equivalência do espaço métrico $L^{p}(\Omega)$ são formadas por funções que diferem apenas num conjunto de medida nula. O fato importante é que $L^{p}(\Omega)$ é um espaço métrico completo para $1 \leq p \leq \infty$, logo a norma em $L^{p}(\Omega)$ o torna um espaço de Banach, e o espaço $L^{2}(\Omega)$ é um espaço de Hilbert com o produto interno

$$
\langle u, v\rangle_{L^{2}}=\int_{\Omega} u v
$$


Mostremos agora que $C_{0}^{\infty}(\Omega)$, o espaço das funções de classe $C^{\infty}$ com suporte compacto em $\Omega$, é denso em $L^{p}(\Omega)$. Isso é feito da seguinte maneira: seja $\varphi \in C_{0}^{\infty}\left(\mathbb{R}^{n}\right)$ uma função não negativa e tal que

$$
\int_{\mathbb{R}^{n}} \varphi(x) d x=1, \quad \operatorname{supp} \varphi \subset \bar{B}(0,1) .
$$

Um exemplo de tal função é dado por

$$
\varphi(x)= \begin{cases}C \exp \left(\frac{-1}{1-|x|^{2}}\right), & |x|<1 \\ 0, & |x| \geq 1\end{cases}
$$

em que $C$ é escolhido de tal modo que $\int_{\mathbb{R}^{n}} \varphi=1$. Para $\varepsilon>0$, a função $\varphi_{\varepsilon}(x)=\varepsilon^{-n} \varphi(x / \varepsilon)$ pertence a $C_{0}^{\infty}\left(\mathbb{R}^{n}\right)$ e $\operatorname{supp} \varphi_{\varepsilon} \subset \bar{B}(0, \varepsilon)$. A função $\varphi_{\varepsilon}$ é chamada de regularizadora e a convolução

$$
u_{\varepsilon}(x)=\varphi_{\varepsilon} * u(x)=u * \varphi_{\varepsilon}(x)=\int_{\mathbb{R}^{n}} \varphi_{\varepsilon}(x-y) u(y) d y
$$

definida para todas as funções $u$ para as quais o lado direito da igualdade acima tem sentido, é chamada de regularização de $u$. O nome regularização é devido ao seguinte resultado:

Teorema 3.3 Se $u \in L^{1}(K)$ para todo compacto $K \subset \mathbb{R}^{n}$, então $u_{\varepsilon} \in C^{\infty}\left(\mathbb{R}^{n}\right)$ e $D^{\alpha}\left(\varphi_{\varepsilon} * u\right)=$ $\left(D^{\alpha} \varphi_{\varepsilon}\right) * u$ para todo multi-índice $\alpha$.

Demonstração: Basta considerar o caso em que $|\alpha|=1$, pois os outros casos seguem por indução. Seja $e_{1}, e_{2}, \ldots, e_{n}$ a base canônica de $\mathbb{R}^{n}$ e observe que

$$
\begin{aligned}
u_{\varepsilon}\left(x+h e_{i}\right)-u_{\varepsilon}(x) & =\int_{\mathbb{R}^{n}} \int_{0}^{h} D_{i} \varphi_{\varepsilon}\left(x-y+t e_{i}\right) u(y) d t d y \\
& =\int_{0}^{h} \int_{\mathbb{R}^{n}} D_{i} \varphi_{\varepsilon}\left(x-y+t e_{i}\right) u(y) d y d t .
\end{aligned}
$$

Como função de $t$, a integral $\int_{\mathbb{R}^{n}} D_{i} \varphi_{\varepsilon}\left(x-y+t e_{i}\right) u(y) d y$ é contínua, logo $D_{i}\left(\varphi_{\varepsilon} * u\right)=\left(D_{i} \varphi_{\varepsilon}\right) * u$.

Teorema 3.4 Se $u \in C\left(\mathbb{R}^{n}\right)$, então $u_{\varepsilon} \rightarrow u$ uniformemente sobre todo compacto $K \in \mathbb{R}^{n}$. 
Demonstração: Seja $K \in \mathbb{R}^{n}$ um compacto fixo. Para todo $\eta>0$, existe $\delta>0$ tal que

$$
|u(x-y)-u(x)|<\eta, \quad \forall x \in K, \quad \forall y \in B(0, \delta) .
$$

Temos

$$
\begin{aligned}
u_{\varepsilon}(x) & -u(x)=\int_{\mathbb{R}^{n}}(u(x-y)-u(x)) \varphi_{\varepsilon}(y) d y= \\
& =\int_{B(0, \varepsilon)}(u(x-y)-u(x)) \varphi_{\varepsilon}(y) d y,
\end{aligned}
$$

e assim, se $\varepsilon<\delta$ e $x \in K$,

$$
\left|u_{\varepsilon}(x)-u(x)\right| \leq \eta \int_{B(0, \varepsilon)} \varphi_{\varepsilon}=\eta
$$

Teorema 3.5 Se $u \in L^{p}\left(\mathbb{R}^{n}\right)$, com $1 \leq p<\infty$, então $u_{\varepsilon} \in L^{p}\left(\mathbb{R}^{n}\right)$, $\left\|u_{\varepsilon}\right\|_{L^{p}} \leq\|u\|_{L^{p}}$ e $u_{\varepsilon} \rightarrow u$ em $L^{p}\left(\mathbb{R}^{n}\right)$ quando $\varepsilon \rightarrow 0$.

Demonstração: Seja $q$ tal que $\frac{1}{p}+\frac{1}{q}=1$. Pela desigualdade de Hölder, temos

$$
\begin{aligned}
\left|u_{\varepsilon}(x)\right| & =\left|\int_{\mathbb{R}^{n}} \varphi_{\varepsilon}(x-y) u(y) d y\right| \\
& \leq\left(\int_{\mathbb{R}^{n}} \varphi_{\varepsilon}(x-y) d y\right)^{1 / q}\left(\int_{\mathbb{R}^{n}} \varphi_{\varepsilon}(x-y)|u(y)|^{p} d y\right)^{1 / p} \\
& =\left(\int_{\mathbb{R}^{n}} \varphi_{\varepsilon}(x-y)|u(y)|^{p} d y\right)^{1 / p} .
\end{aligned}
$$

Logo

$$
\begin{aligned}
\int_{\mathbb{R}^{n}}\left|u_{\varepsilon}(x)\right|^{p} d x & \leq \int_{\mathbb{R}^{n}} \int_{\mathbb{R}^{n}} \varphi_{\varepsilon}(x-y)|u(y)|^{p} d y d x \\
& \leq \int_{\mathbb{R}^{n}} \int_{\mathbb{R}^{n}} \varphi_{\varepsilon}(x-y)|u(y)|^{p} d x d y \\
& =\int_{\mathbb{R}^{n}}|u(y)|^{p} d y,
\end{aligned}
$$


isto é, $\left\|u_{\varepsilon}\right\|_{L^{p}} \leq\|u\|_{L^{p}}$.

Para cada $\eta>0$, seja $v \in C_{0}\left(\mathbb{R}^{n}\right)$ tal que $\|u-v\|_{L^{p}}<\eta$. Como $v$ tem suporte compacto, segue que $\left\|v-v_{\varepsilon}\right\|_{L^{p}}<\eta$ para todo $\varepsilon$ suficientemente pequeno. Temos

$$
\begin{aligned}
\left\|u-u_{\varepsilon}\right\|_{L^{p}} & \leq\|u-v\|_{L^{p}}+\left\|v-v_{\varepsilon}\right\|_{L^{p}}+\left\|v_{\varepsilon}-u_{\varepsilon}\right\|_{L^{p}} \\
& \leq\|u-v\|_{L^{p}}+\left\|v-v_{\varepsilon}\right\|_{L^{p}}+\|v-u\|_{L^{p}}<3 \eta,
\end{aligned}
$$

e portanto $u_{\varepsilon} \rightarrow u$ em $L^{p}\left(\mathbb{R}^{n}\right)$

Corolário 3.3 Seja $\Omega \in \mathbb{R}^{n}$ aberto. Para $1 \leq p<\infty, C_{0}^{\infty}(\Omega)$ é denso em $L^{p}(\Omega)$.

Demonstração: Dada $u \in L^{p}(\Omega)$ e $\eta>0$, seja $v \in C_{0}(\Omega)$ tal que $\|u-v\|_{L^{p}}<\eta$. Seja $v_{1}$ a função definida por

$$
v_{1}(x)= \begin{cases}v(x), & x \in \Omega \\ 0, & x \in \mathbb{R}^{n}-\Omega\end{cases}
$$

Temos $v_{1} \in L^{p}\left(\mathbb{R}^{n}\right)$. Seja $u_{\varepsilon}=\left.\left(\varphi_{\varepsilon} * v_{1}\right)\right|_{\Omega}$. Para $\varepsilon$ suficientemente pequeno, $u_{\varepsilon} \in C_{0}(\Omega)$ e $\| u_{\varepsilon}-$ $v \|_{L^{p}} \stackrel{\varepsilon \rightarrow 0}{\rightarrow} 0$ e portanto, para $\varepsilon$ suficientemente pequeno temos $\left\|u_{\varepsilon}-u\right\|_{L^{p}}<2 \eta$.

\subsection{Os espaços de Sobolev}

Definição 3.3 Seja $1 \leq p \leq \infty$ e $\Omega$ um aberto de $\mathbb{R}^{n}$. O espaço de Sobolev $W^{1, p}(\Omega)$ é definido por

$$
\begin{aligned}
& W^{1, p}(\Omega)=\left\{u \in L^{p}(\Omega): \exists g_{1}, \ldots, g_{n} \in L^{p}(\Omega)\right. \text { tais que } \\
& \left.\int_{\Omega} u \frac{\partial \varphi}{\partial x_{i}}=-\int_{\Omega} g_{i} \varphi \quad \forall \varphi \in C_{0}^{\infty}(\Omega), i=1, \ldots, n\right\} .
\end{aligned}
$$

A motivação para a definição é clara: as funções $g_{i}$ fazem o papel de $\frac{\partial u}{\partial x_{i}}$ (se existisse) na integração por partes. Por isso dizemos que as funções $g_{i}$ são as derivadas fracas de $u$. Denotamos

$$
\frac{\partial u}{\partial x_{i}}=g_{i} \quad \text { e } \quad \nabla u=\left(\frac{\partial u}{\partial x_{1}}, \frac{\partial u}{\partial x_{2}}, \ldots, \frac{\partial u}{\partial x_{n}}\right)
$$


O espaço $W^{1, p}(\Omega)$ é dotado da norma

$$
\|u\|_{W^{1, p}}=\left(\|u\|_{L^{p}}^{p}+\sum_{i=1}^{n}\left\|\frac{\partial u}{\partial x_{i}}\right\|_{L^{p}}^{p}\right)^{1 / p}
$$

ou da norma equivalente

$$
\|u\|_{L^{p}}+\sum_{i=1}^{n}\left\|\frac{\partial u}{\partial x_{i}}\right\|_{L^{p}}
$$

e é um espaço de Banach com esta norma. Denotamos $H^{1}(\Omega)=W^{1,2}(\Omega)$. O espaço $H^{1}(\Omega)$ é um espaço de Hilbert dotado do produto escalar

$$
\langle u, v\rangle_{H^{1}}=\langle u, v\rangle_{L^{2}}+\sum_{i=1}^{n}\left\langle\frac{\partial u}{\partial x_{i}}, \frac{\partial v}{\partial x_{i}}\right\rangle_{L^{2}},
$$

e a norma $\|\cdot\|_{H^{1}}$ associada a este produto escalar é simplesmente $\|\cdot\|_{W^{1,2}}$.

Sabendo que $W^{1, p}(\Omega)$ é um espaço métrico completo, definimos $W_{0}^{1, p}(\Omega)$ como sendo o fecho de $C_{0}^{\infty}(\Omega)$ em $W^{1, p}(\Omega)$, e denotamos $H_{0}^{1}(\Omega)=W_{0}^{1,2}(\Omega)$. O espaço $W_{0}^{1, p}(\Omega)$ é de Banach com a norma induzida de $W^{1, p}(\Omega)$, e o espaço $H_{0}^{1}(\Omega)$ é um espaço de Hilbert com o produto interno de $H^{1}(\Omega)$.

Os espaços de Sobolev $W^{m, p}(\Omega)$ para $m \geq 2$ inteiro são definidos recursivamente:

Definição 3.4 Seja $\Omega$ um aberto de $\mathbb{R}^{n}$. Definimos

$$
W^{m, p}(\Omega)=\left\{u \in W^{m-1, p}(\Omega): \frac{\partial u}{\partial x_{i}} \in W^{m-1, p}(\Omega) \quad \forall i=1,2, \ldots, n\right\} .
$$

A norma em $W^{m, p}(\Omega)$ é dada por

$$
\|u\|_{W^{m, p}}=\left(\int_{\Omega} \sum_{0 \leq|\alpha| \leq m}\left|D^{\alpha} u\right|^{p}\right)^{1 / p},
$$

a qual é equivalente a

$$
\sum_{0 \leq \alpha \leq m}\left\|D^{\alpha} u\right\|_{L^{p}}
$$

em que $D^{\alpha} u$ é a derivada fraca definida pelo multi-índice $\alpha$. Dotado desta norma, o espaço $W^{m, p}(\Omega)$ é um espaço de Banach. Denotamos $H^{m}(\Omega)=W^{m, 2}(\Omega)$, o qual é um espaço de Hilbert com o 
produto interno

$$
\langle u, v\rangle_{H^{m}}=\sum_{0 \leq|\alpha| \leq m}\left\langle D^{\alpha} u, D^{\alpha} v\right\rangle_{L^{2}}
$$

Nos espaços de Sobolev, existem várias desigualdades que nos permitem estimar a norma $L^{p}$ de uma função através da norma de suas derivadas parciais. De fundamental importância é a desigualdade de Sobolev, que enunciaremos para funções no espaço $W_{0}^{1, p}(\Omega)$.

Teorema 3.6 (Desigualdade de Sobolev) Seja $\Omega \subset \mathbb{R}^{n}, n>1$, um aberto. Existe uma constante $C=C(n, p)$ tal que, se $n>p \geq 1$ e $u \in W_{0}^{1, p}(\Omega)$, então

$$
\|u\|_{L^{n p /(n-p)}} \leq C\|\nabla u\|_{L^{p}}
$$

Demonstração: Primeiramente, assuma que $u \in C_{0}^{\infty}(\Omega)$ e que $p=1$. Claramente, para cada $i$, $1 \leq i \leq n$,

$$
|u(x)| \leq \int_{-\infty}^{x_{i}}\left|D_{i} u\left(x_{1}, \ldots, t, \ldots, x_{n}\right)\right| d t
$$

em que $t$ ocupa a $i$-ésima posição no vetor do integrando. Portanto

$$
|u(x)|^{n /(n-1)} \leq\left(\prod_{i=1}^{n} \int_{-\infty}^{+\infty}\left|D_{i} u\right| d x_{i}\right)^{1 /(n-1)} .
$$

Se integrarmos essa desigualdade em relação à primeira variável, $x_{1}$, e então aplicarmos a desigualdade de Hölder com $p_{1}=p_{2}=\ldots=p_{n-1}=n-1$, obtemos

$$
\begin{aligned}
\int_{-\infty}^{+\infty}|u(x)|^{n /(n-1)} d x_{1} \leq & \left(\int_{-\infty}^{+\infty}\left|D_{1} u\left(t, x_{2}, \ldots, x_{n}\right)\right| d t\right)^{1 /(n-1)} \\
& \cdot \int_{-\infty}^{+\infty} \prod_{i=2}^{n}\left(\int_{-\infty}^{+\infty}\left|D_{i} u\right| d x_{i}\right)^{1 /(n-1)} d x_{1} \\
\leq & \left(\int_{-\infty}^{+\infty}\left|D_{1} u\left(t, x_{2}, \ldots, x_{n}\right)\right| d t\right)^{1 /(n-1)} \\
& \cdot \prod_{i=2}^{n}\left(\int_{-\infty}^{+\infty} \int_{-\infty}^{+\infty}\left|D_{i} u\right| d x_{i} d x_{1}\right)^{1 /(n-1)} .
\end{aligned}
$$


Continuando esse procedimento e integrando (3.1) com relação a cada variável, obtemos

$$
\int_{\mathbb{R}^{n}}|u(x)|^{n /(n-1)} d x \leq \prod_{i=1}^{n}\left(\int_{\mathbb{R}^{n}}\left|D_{i} u\right| d x\right)^{1 /(n-1)}
$$

e, usando a desigualdade entre as médias aritmética e geométrica, isto é, que

$$
\left(\prod_{j=1}^{n} a_{j}\right)^{1 / n} \leq \frac{1}{n} \sum_{j=1}^{n} a_{j}, \quad a_{j} \geq 0
$$

temos

$$
\|u\|_{L^{n /(n-1)}} \leq \prod_{i=1}^{n}\left(\int_{\mathbb{R}^{n}}\left|D_{i} u\right| d x\right)^{1 / n} \leq \frac{1}{n} \int_{\mathbb{R}^{n}} \sum_{i=1}^{n}\left|D_{i} u\right| d x \leq \frac{\sqrt{n}}{n}\|\nabla u\|_{L^{1}},
$$

o que demonstra o caso $p=1$ quando $u \in C_{0}^{\infty}(\Omega)$. O resultado quando $p>1$ pode ser obtido de (3.2) substituindo $|u|$ por potências de $|u|$ : se $p>1$, seja $q$ tal que $\frac{1}{p}+\frac{1}{q}=1$. Para todo $\ell>1$, temos

$$
\begin{aligned}
\left\|\left|u^{\ell}\right|\right\|_{L^{n /(n-1)}} & \leq \frac{\sqrt{n}}{n} \int_{\mathbb{R}^{n}}\left|\nabla\left(|u|^{\ell}\right)\right| d x \\
& \leq \frac{\ell \sqrt{n}}{n} \int_{\mathbb{R}^{n}}|u|^{\ell-1}|\nabla u| d x \\
& \leq \frac{\ell \sqrt{n}}{n}\left\||u|^{\ell-1}\right\|_{L^{q}}\|\nabla u\|_{L^{p}},
\end{aligned}
$$

pela desigualdade de Hölder. Para $\ell=\frac{(n-1) p}{n-p}$, essa desigualdade fica

$$
\begin{aligned}
\left(\int_{\Omega}\left(|u|^{\frac{(n-1) p}{n-p}}\right)^{\frac{n}{n-1}}\right)^{\frac{n-1}{n}} & \leq \frac{(n-1) p \sqrt{n}}{n(n-p)}\left(\int_{\Omega}\left(|u|^{\frac{n(p-1)}{n-p}}\right)^{\frac{p}{p-1}}\right)^{\frac{p-1}{p}}\|\nabla u\|_{L^{p}} \Longleftrightarrow \\
\left(\int_{\Omega}|u|^{\frac{n p}{n-p}}\right)^{\frac{n-1}{n}} & \leq \frac{(n-1) p \sqrt{n}}{n(n-p)}\left(\int_{\Omega}|u|^{\frac{n p}{n-p}}\right)^{\frac{p-1}{p}}\|\nabla u\|_{L^{p}} \Longleftrightarrow \\
\left(\int_{\Omega}|u|^{\frac{n p}{n-p}}\right)^{\frac{n-p}{n p}} & \leq \frac{(n-1) p \sqrt{n}}{n(n-p)}\|\nabla u\|_{L^{p}} \Longleftrightarrow \\
\|u\|_{L^{n p /(n-p)}} & \leq \frac{(n-1) p \sqrt{n}}{n(n-p)}\|\nabla u\|_{L^{p}},
\end{aligned}
$$


o que prova o teorema no caso em que $n>p \geq 1$ e $u \in C_{0}^{\infty}(\Omega)$. Agora, seja $u \in W_{0}^{1, p}(\Omega)$ e seja $\left(u_{k}\right)_{k \geq 1}$ uma seqüência de funções em $C_{0}^{\infty}(\Omega)$ que convergem para $u$ em $W_{0}^{1, p}(\Omega)$. Aplicando a desigualdade para $u_{i}-u_{j}$, obtemos

$$
\left\|u_{i}-u_{j}\right\|_{L^{n p /(n-p)}} \leq C\left\|u_{i}-u_{j}\right\|_{W^{1, p}}
$$

$\operatorname{logo} u_{k} \rightarrow u$ em $L^{n p /(n-p)}(\Omega)$ e o resultado segue.

O seguinte corolário é conseqüencia imediata da desigualdade de Sobolev:

Corolário 3.4 Seja $\Omega \subset \mathbb{R}^{n}$ um aberto, $n>2$. Existe uma constante $C=C(n)$ tal que, se $u \in H_{0}^{1}(\Omega)$, então

$$
\|u\|_{L^{2 n /(n-2)}} \leq C\|u\|_{H^{1}}
$$

Em seguida, podemos demonstrar uma versão restrita do Teorema de Rellich-Kondrachov:

Teorema 3.7 (Teorema de Rellich-Kondrachov) Seja $\Omega \subset \mathbb{R}^{n}$ um aberto limitado. Então, se $n>2$, a inclusão de $H_{0}^{1}(\Omega)$ em $L^{q}(\Omega)$ é compacta para todo $q$ satisfazendo $1 \leq q<\frac{2 n}{n-2}$.

Demonstração: Seja $B \subset H_{0}^{1}(\Omega)$ um conjunto limitado. Mostraremos que $\bar{B}$ é compacto em $L^{q}(\Omega)$. Como $C_{0}^{\infty}(\Omega)$ é denso em $H_{0}^{1}(\Omega)$, podemos assumir sem perda de generalidade que $B \subset C_{0}^{\infty}(\Omega)$. Por conveniência, também iremos assumir que $\|u\|_{H^{1}} \leq 1$ para todo $u \in B$.

Para $\varepsilon>0$, seja $u_{\varepsilon}$ a regularização de $u$. Isto é, $u_{\varepsilon}=u * \varphi_{\varepsilon}$, em que $\varphi_{\varepsilon}$ é a função regularizadora. Se $u \in B$, temos

$$
\begin{aligned}
\left|u_{\varepsilon}(x)\right| & \leq \int_{B(0, \varepsilon)}|u(x-y)| \varphi_{\varepsilon}(y) d y \\
& \leq \varepsilon^{-n}\|u\|_{L^{1}} \sup \left\{\varphi(y): y \in \mathbb{R}^{n}\right\} \\
& \leq \varepsilon^{-n} \sup \left\{\varphi(y): y \in \mathbb{R}^{n}\right\}
\end{aligned}
$$


e

$$
\begin{aligned}
\left|D u_{\varepsilon}(x)\right| & \leq \int_{B(0, \varepsilon)}|u(x-y)|\left|D \varphi_{\varepsilon}(y)\right| d y \\
& \leq \varepsilon^{-n-1}\|u\|_{L^{1}} \sup \left\{|D \varphi(y)|: y \in \mathbb{R}^{n}\right\} \\
& \leq \varepsilon^{-n-1} \sup \left\{|D \varphi(y)|: y \in \mathbb{R}^{n}\right\} .
\end{aligned}
$$

Portanto, sendo $B_{\varepsilon}=\left\{u_{\varepsilon}: u \in B\right\}$, temos que $B_{\varepsilon}$ é um subconjunto uniformemente limitado e eqüicontínuo de $C(\bar{\Omega})$. Pelo Teorema de Ascoli-Arzela, segue que $B_{\varepsilon}$ tem fecho compacto em $C(\bar{\Omega})$, e conseqüentemente tem fecho compacto em $L^{1}(\Omega)$. Observe também que, para $\gamma(t)=t(x-y)+$ $(1-t) x=x-t y$

$$
\begin{aligned}
\left|u(x)-u_{\varepsilon}(x)\right| & \leq \int_{B(0, \varepsilon)}|u(x)-u(x-y)| \varphi_{\varepsilon}(y) d y \\
& \leq \int_{B(0, \varepsilon)} \int_{0}^{1}\left|D u \circ \gamma(t) \cdot \gamma^{\prime}(t)\right| \varphi_{\varepsilon}(y) d t d y \\
& \leq \int_{B(0, \varepsilon)} \int_{0}^{1}|D u(x-t y)||y| \varphi_{\varepsilon}(y) d t d y .
\end{aligned}
$$

Conseqüentemente, pelo Teorema de Fubini temos

$$
\begin{aligned}
\int_{\mathbb{R}^{n}}\left|u(x)-u_{\varepsilon}(x)\right| d x & \leq \int_{B(0, \varepsilon)} \int_{0}^{1} \int_{\mathbb{R}^{n}}|D u(x-t y)||y| \varphi_{\varepsilon}(y) d x d t d y \\
& \leq \varepsilon \int_{\Omega}|D u| d x \leq \varepsilon
\end{aligned}
$$

Portanto $B$ está contido em uma $\varepsilon$-vizinhança de $B_{\varepsilon}$ em $L^{1}(\Omega)$. Como o fecho de $B_{\varepsilon}$ é compacto em $L^{1}(\Omega), B_{\varepsilon}$ é totalmente limitado. Ou seja, para todo $r>0$ existe um número finito de bolas de raio $r$ em $L^{1}(\Omega)$ cuja união contém $B_{\varepsilon}$. Logo $B$ é totalmente limitado e portanto $\bar{B}$ é compacto em $L^{1}(\Omega)$, o que demonstra o teorema para $q=1$.

Se $1 \leq q<2 n /(n-2)$, o Corolário 3.4 e o Corolário 3.1 nos dão, para $\lambda=\frac{1 / q-(n-2) /(2 n)}{1-(n-2) /(2 n)}$,

$$
\|u\|_{L^{q}} \leq\|u\|_{L^{1}}^{\lambda}\|u\|_{L^{2 n /(n-2)}}^{1-\lambda} \leq C\|u\|_{L^{1}}^{\lambda}\|u\|_{H^{1}}^{1-\lambda}
$$


o que implica que conjuntos limitados em $H_{0}^{1}(\Omega)$ são totalmente limitados em $L^{q}(\Omega)$ e portanto com fecho compacto.

Como conseqüência dos resultados anteriores, obtemos a desigualdade de Poincaré:

Teorema 3.8 (Desigualdade de Poincaré) Suponha que $\Omega \subset \mathbb{R}^{n}$ é um aberto limitado, $n>2$. Existe uma constante $C$ (dependendo de $\Omega$ ) tal que

$$
\|u\|_{L^{2}} \leq C\|\nabla u\|_{L^{2}}
$$

para toda $u \in H_{0}^{1}(\Omega)$.

Demonstração: Se $n>2$, então $\frac{2 n}{n-2}>2$, e portanto a inclusão $i: H_{0}^{1}(\Omega) \rightarrow L^{2}(\Omega)$ é compacta, pelo teorema de Rellich-Kondrachov. Seja então

$$
\begin{aligned}
K & =\left\{u \in H_{0}^{1}(\Omega): \sqrt{\|u\|_{L^{2}}^{2}+\|\nabla u\|_{L^{2}}^{2}}=1\right\} \\
& =\left\{u \in H_{0}^{1}(\Omega):\|u\|_{H^{1}}=1\right\}
\end{aligned}
$$

O conjunto $K$ possui fecho compacto em $L^{2}(\Omega)$. Mas $K$ é fechado em $L^{2}(\Omega)$, isto é, $\bar{K}=K$ em $L^{2}(\Omega)$. De fato, suponha que a seqüência $\left(u_{k}\right)_{k \geq 1}$ de elementos de $K$ converge para $u$ em $L^{2}(\Omega)$. Esta seqüência é limitada em $H_{0}^{1}(\Omega)$, logo possui uma subseqüência, também denotada por $\left(u_{k}\right)_{k \geq 1}$, tal que $u_{k} \rightarrow u^{\prime} \in H_{0}^{1}(\Omega)$. Mas então, como a inclusão de $H_{0}^{1}(\Omega)$ em $L^{2}(\Omega)$ é compacta, temos $u_{k} \rightarrow u^{\prime}$ em $L^{2}(\Omega)$. Portanto $u^{\prime}=u$, ou seja, $u \in H_{0}^{1}(\Omega)$.

Definimos

$$
\begin{aligned}
f: K & \rightarrow \mathbb{R} \\
u & \mapsto\|u\|_{L^{2}} .
\end{aligned}
$$

A função $f$ é contínua e $K$ é compacto, $\log$ o $f$ assume um valor máximo. Seja $C$ este máximo. Afirmamos que $C<1$. Claramente temos $C \leq 1$. Supondo que $C=1$, existe $u \in K$ tal que $\|u\|_{L^{2}}=1$, ou seja, $\|\nabla u\|_{L^{2}}=0$. Pela desigualdade de Sobolev, $\|u\|_{L^{2 n /(n-2)}}=0 \Rightarrow u=0$ quase sempre em $\Omega$, o que é um absurdo, pois $\|u\|_{L^{2}}=1$. Logo, de fato, temos $C<1$. 
A norma de $\|i\|$ é dada por

$$
\|i\|=\sup _{u \neq 0} \frac{\|u\|_{L^{2}}}{\|u\|_{H^{1}}}<1
$$

pois $C<1$. Portanto, se $u \in H_{0}^{1}(\Omega)$,

$$
\begin{gathered}
\|u\|_{L^{2}} \leq\|i\| \sqrt{\|u\|_{L^{2}}^{2}+\|\nabla u\|_{L^{2}}^{2}} \Longleftrightarrow \\
\|u\|_{L^{2}} \leq \frac{\|i\|}{\sqrt{1-\|i\|^{2}}}\|\nabla u\|_{L^{2}} .
\end{gathered}
$$




\section{Capítulo 4}

\section{O operador $-\Delta+\alpha \chi_{\mathbf{D}}$}

\subsection{Membranas compostas ótimas}

Recordemos que o ponto de partida para o nosso estudo é o seguinte problema: é dada uma membrana, cuja fronteira e massa total estão fixadas, e cuja densidade é variável. Queremos descobrir qual distribuição de densidade da membrana torna a freqüência fundamental desta a menor possível, isto é, queremos a distribuição de densidade que nos dá uma membrana com o tom mais grave. A formulação matemática para este problema é a seguinte: a fronteira da membrana é $\partial \Omega$, em que $\Omega \subset \mathbb{R}^{n}$ é um conjunto aberto, limitado e conexo. São dados dois números reais positivos $\rho_{1}$ e $\rho_{2}$ satisfazendo $\rho_{1}<\rho_{2}$, que são respectivamente os limites inferior e superior para a densidade de nossa membrana. A massa total da membrana, também fixada, é um número $M \in\left[\rho_{1}|\Omega|, \rho_{2}|\Omega|\right], M>0$. Consideramos funções mensuráveis $\rho$ (que representam a densidade) satisfazendo

$$
\rho_{1} \leq \rho \leq \rho_{2}, \quad \int_{\Omega} \rho=M
$$

com o objetivo de encontrar $\rho$ e $u$ que realizem o mínimo em

$$
\Theta\left(\rho_{1}, \rho_{2}, M\right)=\inf _{\rho} \inf _{u \in H_{0}^{1}(\Omega)} \frac{\int_{\Omega}|\nabla u|^{2}}{\int_{\Omega} \rho u^{2}} .
$$


O problema de autovalores correspondente é

$$
(*)_{\Omega, \rho}= \begin{cases}-\Delta u=\lambda \rho u & \text { em } \Omega \\ u=0 & \text { em } \partial \Omega\end{cases}
$$

no qual o nosso objetivo é minimizar o valor do primeiro autovalor $\lambda_{1}$, o qual é dado pela equação (4.1). Se a função $\rho$ minimiza o valor de $\lambda_{1}$ e $u$ satisfaz $(*)_{\Omega, \rho}$, dizemos que o par $(u, \rho)$ é ótimo para o problema $(*)_{\Omega, \rho}$, com parâmetros $\left(\rho_{1}, \rho_{2}, M\right)$.

Podemos também considerar um outro problema (o Teorema 4.2 relacionará ambos): são dados um conjunto $\Omega \subset \mathbb{R}^{n}$ aberto, limitado e conexo, e números $\alpha>0, A \in[0,|\Omega|]$. Para qualquer conjunto mensurável $D$, seja $\chi_{D}$ sua função característica e $\lambda_{\Omega}(\alpha, D)$ o menor autovalor do problema

$$
(*)_{\Omega, \alpha, D}= \begin{cases}-\Delta u+\alpha \chi_{D} u=\lambda u & \text { em } \Omega \\ u=0 & \text { em } \partial \Omega .\end{cases}
$$

A caracterização variacional para $\lambda_{\Omega}(\alpha, D)$ é

$$
\lambda_{\Omega}(\alpha, D)=\inf _{u \in H_{0}^{1}(\Omega)} \frac{\int_{\Omega}|\nabla u|^{2}+\alpha \int_{\Omega} \chi_{D} u^{2}}{\int_{\Omega} u^{2}} .
$$

Definimos

$$
\Lambda_{\Omega}(\alpha, A)=\inf \left\{\lambda_{\Omega}(\alpha, D): D \subset \Omega,|D|=A\right\}
$$

Qualquer $D$ que realize o mínimo em (4.2) é chamado de configuração ótima para os parâmetros $(\alpha, A)$. Se $D$ é uma configuração ótima e $u$ satisfaz $(*)_{\Omega, \alpha, D}$, chamamos o par $(u, D)$ de ótimo para o problema $(*)_{\Omega, \alpha, D}$, com parâmetros $(\alpha, A)$. Não consideraremos $\Omega$ um parâmetro para o problema, já que $\Omega$ é suposto fixo.

Como a função $\chi_{D}$ não é contínua, a primeira equação de $(*)_{\Omega, \alpha, D}$ deve ser entendida num sentido fraco, que definimos a partir de agora. Multiplicando a primeira equação de $(*)_{\Omega, \alpha, D}$ por $v \in H_{0}^{1}(\Omega)$ e integrando sobre $\Omega$, temos, integrando por partes,

$$
\begin{gathered}
\int_{\Omega}(-\Delta u) v+\alpha \chi_{D} u v=\lambda \int_{\Omega} u v \Rightarrow \\
\int_{\Omega} \nabla u \cdot \nabla v+\alpha \chi_{D} u v=\lambda \int_{\Omega} u v .
\end{gathered}
$$


Por essa razão, dizemos que $u \in H_{0}^{1}(\Omega)$ é solução fraca de $(*)_{\Omega, \alpha, D}$ quando

$$
\int_{\Omega} \nabla u \cdot \nabla v+\alpha \chi_{D} u v=\lambda \int_{\Omega} u v
$$

para toda $v \in H_{0}^{1}(\Omega)$.

Antes de relacionarmos os problemas $(*)_{\Omega, \rho}$ e $(*)_{\Omega, \alpha, D}$, o seguinte teorema estabelece a existência e as propriedades básicas das soluções de $(*)_{\Omega, \alpha, D}$.

Teorema 4.1 ([2]) Para todo $\alpha>0$ e todo $A \in[0,|\Omega|]$ existe um par ótimo para $(*)_{\Omega, \alpha, D}$. Além disso, qualquer par ótimo $(u, D)$ possui as seguintes propriedades:

1. $u \in C^{1, \beta}(\Omega) \cap H^{2}(\Omega) \cap C^{\gamma}(\bar{\Omega})$ para algum $\gamma>0$ e todo $\beta<1$.

2. Dé um subnível de $u$, isto é, existe $c \geq 0$ tal que

$$
D=\{x \in \Omega: u(x) \leq c\}
$$

\section{Demonstração:}

1. A regularidade das soluções são válidas para equações do tipo

$$
-\Delta u+\zeta u=0
$$

com $\zeta$ limitada [12, Teorema 8.29 e Corolário 8.36].

Para mostrar a existência das soluções, fixe $\alpha$ e $A$, e escreva, por simplicidade, $\Lambda=\Lambda_{\Omega}(\alpha, A)$, $\lambda(D)=\lambda_{\Omega}(\alpha, D)$. Seja $\left(D_{k}\right)_{k \geq 1}$ uma seqüência minimizante, isto é, $\lambda\left(D_{k}\right) \rightarrow \Lambda$ quando $k \rightarrow \infty$. Seja $u_{k} \in H_{0}^{1}(\Omega)$ a autofunção de $-\Delta+\alpha \chi_{D_{k}}$ normalizada em $L^{2}(\Omega)$. Como $\left(\lambda\left(D_{k}\right)\right)_{k \geq 1}$ é uma seqüencia limitada, a seqüência $\left(u_{k}\right)_{k \geq 1}$ é limitada em $H_{0}^{1}(\Omega)$. Além disso, $\left(\chi_{D_{k}}\right)_{k \geq 1}$ é limitada em $L^{2}(\Omega)$. Logo podemos escolher subseqüências, novamente denotadas $\left(D_{k}\right)_{k \geq 1}$ e $\left(u_{k}\right)_{k \geq 1}$, tais que $u_{k} \rightarrow u$ em $H_{0}^{1}(\Omega)$ e $\chi_{D_{k}} \rightarrow \eta$ em $L^{2}(\Omega)$. Isto implica que $u_{k} \rightarrow u$ em $L^{2}(\Omega), \chi_{D_{k}} u_{k} \rightarrow \eta u$ em $L^{2}(\Omega)$, e $\int_{\Omega} \eta=A$. Tomando limites na equação fraca

$$
\int_{\Omega} \nabla u_{k} \cdot \nabla v+\alpha \int_{\Omega} \chi_{D_{k}} u_{k} v=\lambda\left(D_{k}\right) \int_{\Omega} u_{k} v \quad \forall v \in H_{0}^{1}(\Omega)
$$

obtemos

$$
-\Delta u+\alpha \eta u=\Lambda u \text {. }
$$


Temos $0 \leq \eta \leq 1$, já que $0 \leq \chi_{D_{k}} \leq 1$ para todo $k \geq 1$ e a convergência fraca preserva desigualdades pontuais quase sempre. Portanto $u$ tem a regularidade especificada no item 1 .

Resta mostrar que podemos substituir $\eta$ por uma função característica. Como $\int_{\Omega} u^{2}=1$, a equação (4.4) mostra que

$$
\int_{\Omega}|\nabla u|^{2}+\alpha \int_{\Omega} \eta u^{2}=\Lambda
$$

Mas o problema de minimização

$$
\inf \left\{\int_{\Omega} \eta u^{2}: 0 \leq \eta \leq 1, \int_{\Omega} \eta=A\right\}
$$

possui uma solução $\eta=\chi_{D}$, em que $D$ é qualquer subconjunto de $\Omega$ que satisfaz $|D|=A$ e

$$
\begin{array}{r}
\{x \in \Omega: u(x)<c\} \subset D \subset\{x \in \Omega: u(x) \leq c\}, \\
c=\sup \{s \in \mathbb{R}:|\{x \in \Omega: u(x)<s\}|<A\} .
\end{array}
$$

Portanto temos

$$
\int_{\Omega}|\nabla u|^{2}+\alpha \int_{\Omega} \chi_{D} u^{2} \leq \Lambda
$$

Mas, pela definição de $\Lambda$ como um mínimo, a desigualdade acima deve ser uma igualdade, e $(u, D)$ é solução.

2. Seja $(u, D)$ uma solução. É claro que (4.5) deve valer quase sempre: caso contrário, poderíamos reduzir $\int_{D} u^{2}$ ao mover uma parte de $D$ de $\{x \in \Omega: u(x)>c\}$ para $\{x \in \Omega: u(x) \leq c\}$. Seja $N_{s}=\{x \in \Omega: u(x)=s\}$ para qualquer $s>0$. Usando o Lema 7.7 de [12] duas vezes, vemos que $\Delta u=0$ quase sempre em $N_{s}$ (uma vez que $u$ é constante em $N_{s}$; lembre que $u \in H^{2}(\Omega)$ ). Assim,

$$
\left(\Lambda-\alpha \chi_{D}\right) u=0 \quad \text { quase sempre em } N_{s} .
$$

Como $u>0$ e $\Lambda>0$, isto mostra que $D^{c} \cap N_{s}$ tem medida zero. Basta então tomar $s=c$.

A relação entre os problemas $(*)_{\Omega, \rho}$ e $(*)_{\Omega, \alpha, D}$ é dada pelo teorema a seguir, que mostra, entre outras coisas, que a função de distribuição de densidade que minimiza o primeiro autovalor do problema $(*)_{\Omega, \rho}$ não é contínua. 
Teorema 4.2 ([2]) Sejam $\Omega, 0<\rho_{1}<\rho_{2}<\infty$ e $M \in\left[\rho_{1}|\Omega|, \rho_{2}|\Omega|\right] \cap \mathbb{R}_{+}^{*}$ dados.

1. A função de distribuição de densidade $\rho$ que minimiza o primeiro autovalor $\lambda_{1}$ do problema $(*)_{\Omega, \rho}$ é da forma

$$
\rho=\rho_{D}=\rho_{1} \chi_{D}+\rho_{2} \chi_{D^{c}}
$$

em que $D$ é um subconjunto (fechado) de $\Omega$ e $D^{c}$ seu complementar. Além disso, se u é a primeira autofunção associada a $\lambda_{1}, D$ é um subnível de $u$, isto é, existe $c>0$ tal que

$$
D=\{x \in \Omega: u(x) \leq c\}
$$

2. O par $\left(u, \rho_{D}\right)$ é ótimo para o problema $(*)_{\Omega, \rho}$, com parâmetros $\left(\rho_{1}, \rho_{2}, M\right)$ se, e somente se, o par $(u, D)$ é ótimo para o problema de autovalores $(*)_{\Omega, \alpha, D}$, com parâmetros $(\alpha, A)$ dados por $\alpha=\left(\rho_{2}-\rho_{1}\right) \lambda_{1}$ e $A=\frac{\rho_{2}|\Omega|-M}{\rho_{2}-\rho_{1}}$. Além disso, temos também $\Lambda(\alpha, A)=\rho_{2} \lambda_{1}$.

\section{Demonstração:}

1. O primeiro autovalor $\lambda_{1}$ de $(*)_{\Omega, \rho}$ com parâmetros $\left(\rho_{1}, \rho_{2}, M\right)$ é dado por

$$
\lambda_{1}=\Theta\left(\rho_{1}, \rho_{2}, M\right)=\inf _{\substack{\rho_{1} \leq \rho \leq \rho_{2} \\ \int_{\Omega} \rho=M}} \inf _{u \in H_{0}^{1}(\Omega)} \frac{\int_{\Omega}|\nabla u|^{2}}{\int_{\Omega} \rho u^{2}} .
$$

Para $u$ fixo, o maior valor de $\int_{\Omega} \rho u^{2}$ para $\rho_{1} \leq \rho \leq \rho_{2}$ e $\int_{\Omega} \rho=M$ é obtido quando $\rho=$ $\rho_{1} \chi_{D}+\rho_{2} \chi_{D^{c}}$, em que $D$ é um subnível de $u$ de medida adequada, de modo a termos $\int_{\Omega} \rho=M$. A justificativa para tal fato é análoga à do teorema anterior, no qual minimizamos o valor de $\int_{\Omega} \eta u^{2}$, em que $\eta$ era uma função com imagem contida em $[0,1]$ e $\int_{\Omega} \eta=A$.

2. Seja $\Theta \in \mathbb{R}$. Temos

$$
\begin{gathered}
-\Delta u=\Theta \rho_{D} u=\Theta\left(\rho_{1} \chi_{D}+\rho_{2} \chi_{D^{c}}\right) u \Longleftrightarrow \\
\Longleftrightarrow-\Delta u+\left(\rho_{2}-\rho_{1}\right) \Theta \chi_{D} u=\rho_{2} \Theta u
\end{gathered}
$$

Se $\left(u, \rho_{D}\right)$ é par ótimo para o problema $(*)_{\Omega, \rho}$, então satisfaz (4.6) com $\Theta=\Theta\left(\rho_{1}, \rho_{2}, M\right)$ e a equação (4.7) mostra que $\Lambda(\alpha, A) \leq \rho_{2} \Theta\left(\rho_{1}, \rho_{2}, M\right)$ para $\alpha=\left(\rho_{2}-\rho_{1}\right) \Theta\left(\rho_{1}, \rho_{2}, M\right)$.

Por outro lado, se $(u, D)$ é par ótimo para o problema $(*)_{\Omega, \alpha, D}$ com parâmetros $(\alpha, A)$ dados por $\alpha=\left(\rho_{2}-\rho_{1}\right) \Theta\left(\rho_{1}, \rho_{2}, M\right)$ e $A=\frac{\rho_{2}|\Omega|-M}{\rho_{2}-\rho_{1}}$, então a equação (4.7) vale com $\rho_{2} \Theta$ substituído 
por $\Lambda=\Lambda(\alpha, A)$, e portanto, no lugar de (4.6), obtemos

$$
-\Delta u=\Theta \rho_{D} u+\left(\Lambda-\rho_{2} \Theta\right) u,
$$

em que $\Theta=\Theta\left(\rho_{1}, \rho_{2}, M\right)$. Multiplicando por $u$ e integrando sobre $\Omega$, ficamos com

$$
\int_{\Omega}|\nabla u|^{2}=\Theta \int_{\Omega} \rho_{D} u^{2}+\left(\Lambda-\rho_{2} \Theta\right) \int_{\Omega} u^{2}
$$

Pela definição de $\Theta$, temos $\int_{\Omega}|\nabla u|^{2} \geq \Theta \int_{\Omega} \rho_{D} u^{2}, \operatorname{logo} \Lambda \geq \rho_{2} \Theta$ e portanto $\Lambda(\alpha, A)=$ $\rho_{2} \Theta\left(\rho_{1}, \rho_{2}, M\right)$.

A membrana que resulta do teorema acima é chamada de membrana composta ótima. Ela é feita de dois materiais de densidades distintas. O que fazemos então é reverter a situação e considerar membranas cujas distribuições de massa são desse tipo e estudar o problema variacional associado para o primeiro autovalor. Neste novo problema variacional, a condição da massa total fixada se traduz numa condição para a medida de $D$, pois, como vimos,

$$
|D|=\frac{\rho_{2}|\Omega|-M}{\rho_{2}-\rho_{1}} .
$$

Consideramos, então, o problema de procurar $D$ de volume fixado para o qual $\lambda_{\Omega}(\alpha, D)$ é mínimo, isto é, que realiza o ínfimo

$$
\Lambda_{\Omega}(\alpha, A)=\inf \left\{\lambda_{\Omega}(\alpha, D): D \subset \Omega,|D|=A\right\}, \quad(A \in[0,|\Omega|]) .
$$

Antes, porém, de estudarmos este novo problema variacional, vamos demonstrar certas propriedades do operador $-\Delta+\alpha \chi_{D}$ que usamos livremente até o momento. O nosso primeiro objetivo é demonstrar que os autovalores de $(*)_{\Omega, \alpha, D}$ são positivos e formam uma seqüência que tende a $+\infty$. Em seguida, mostraremos a caracterização variacional do primeiro autovalor (a qual já utilizamos) e também mostraremos que o primeiro autovalor é simples. Além disso, temos também que mostrar que a autofunção correspondente ao primeiro autovalor pode ser tomada positiva e de norma $1 \mathrm{em}$ $L^{2}(\Omega)$. Isto nos leva naturalmente ao estudo do operador $-\Delta+\alpha \chi_{D}$, para $\alpha$ e $D$ fixados. 


\subsection{Estudo do operador $-\Delta+\alpha \chi_{D}$}

Seja $\Omega \subset \mathbb{R}^{n}$ um aberto limitado, $n>2$. Recordemos que $H_{0}^{1}(\Omega)$ é um espaço de Hilbert com o produto interno definido por $\langle u, v\rangle_{H^{1}}=\int_{\Omega} u v+\nabla u \cdot \nabla v$ e norma associada $\|u\|_{H^{1}}=\sqrt{\langle u, u\rangle_{H^{1}}}$. Podemos considerar também outros produtos internos em $H_{0}^{1}(\Omega)$, como o definido por $\langle u, v\rangle_{H^{1}}^{*}=$ $\int_{\Omega} \nabla u \cdot \nabla v$, cuja norma associada é $\|u\|_{H^{1}}^{*}=\sqrt{\langle u, u\rangle_{H^{1}}^{*}}=\|\nabla u\|_{L^{2}}$.

Proposição 4.1 Seja a: $H_{0}^{1}(\Omega) \times H_{0}^{1}(\Omega) \rightarrow \mathbb{R}$ a forma bilinear definida por

$$
a(u, v)=\int_{\Omega} \nabla u \cdot \nabla v+\alpha \chi_{D} u v .
$$

Esta forma bilinear define um produto interno em $H_{0}^{1}(\Omega)$, que denotamos por $\langle\cdot, \cdot\rangle_{a}$. Se sua norma correspondente é denotada por $\|\cdot\|_{a}$, então as normas $\|\cdot\|_{H^{1}}^{*},\|\cdot\|_{H^{1}}$ e $\|\cdot\|_{a}$ são equivalentes.

Demonstração: É imediato verificar que $\langle u, v\rangle_{a}=a(u, v)$ define um produto interno em $H_{0}^{1}(\Omega)$. Verifiquemos em primeiro lugar que as normas $\|\cdot\|_{a}$ e $\|\cdot\|_{H^{1}}^{*}$ são equivalentes. Claramente temos $\|u\|_{a} \geq\|u\|_{H^{1}}^{*}$. Por outro lado,

$$
\begin{aligned}
\|u\|_{a}^{2} & =\int_{\Omega}|\nabla u|^{2}+\alpha \chi_{D} u^{2} \leq \int_{\Omega}|\nabla u|^{2}+\alpha \int_{\Omega}|u|^{2} \\
& \leq \int_{\Omega}|\nabla u|^{2}+C \alpha|\nabla u|^{2}=(C \alpha+1)\left(\|u\|_{H^{1}}^{*}\right)^{2},
\end{aligned}
$$

isto é, $\|u\|_{a} \leq \sqrt{(C \alpha+1)}\|u\|_{H^{1}}$. Na segunda desigualdade acima, utilizamos a desigualdade de Poincaré. Segue que as normas $\|\cdot\|_{a}$ e $\|\cdot\|_{H^{1}}^{*}$ são equivalentes. De maneira análoga mostra-se que

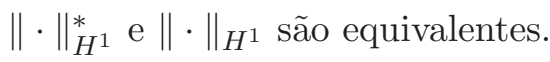

Em particular, a proposição anterior nos permite concluir que o espaço $H_{0}^{1}(\Omega)$ é um espaço de Hilbert quando munido do produto interno $\langle\cdot, \cdot\rangle_{a}$. 
Para um $u \in H_{0}^{1}(\Omega)$ fixado, a aplicação $v \stackrel{F}{\mapsto} \int_{\Omega} u v$ é um funcional linear limitado em $H_{0}^{1}(\Omega)$, pois pelas desigualdades de Cauchy-Schwarz e Poincaré temos

$$
\begin{aligned}
|F v|=\left|\int_{\Omega} u v\right| & \leq \int_{\Omega}\left|u\|v \mid \leq\| u\left\|_{L^{2}}\right\| v \|_{L^{2}}\right. \\
& \leq\left(C\|u\|_{L^{2}}\right)\|\nabla v\|_{L^{2}}=\left(C\|u\|_{L^{2}}\right)\|v\|_{H^{1}}^{*}
\end{aligned}
$$

Logo, pelo teorema de representação de Riesz, existe um elemento em $H_{0}^{1}(\Omega)$, que denotaremos por $Q u$, tal que

$$
\langle Q u, v\rangle_{a}=\int_{\Omega} u v
$$

O operador $Q: H_{0}^{1}(\Omega) \rightarrow H_{0}^{1}(\Omega)$ é linear, simétrico e limitado (pois $\|Q u\|=\|F\| \leq C\|u\|_{L^{2}} \leq$ $C^{\prime}\|u\|_{H^{1}}^{*}$ ). Além disso, $Q$ é compacto. De fato, seja $\left(u_{k}\right)_{k \geq 1}$ uma seqüência limitada em $H_{0}^{1}(\Omega)$. Passando a uma subseqüência, se necessário, podemos assumir que $u_{k} \rightarrow u$ em $H_{0}^{1}(\Omega), u_{k} \rightarrow u$ em $L^{s}(\Omega)$, para $s<\frac{2 n}{n-2}$, pelo teorema de Rellich-Kondrachov. Usando a equação (4.8) com $u_{k}-u$ no lugar de $u$ e $Q u_{k}-Q u$ no lugar de $v$, obtemos

$$
\left\langle Q u_{k}-Q u, Q u_{k}-Q u\right\rangle_{a}=\int_{\Omega}\left(u_{k}-u\right)\left(Q u_{k}-Q u\right)=\int_{\Omega} 1\left(u_{k}-u\right)\left(Q u_{k}-Q u\right) .
$$

Quando $n>2, \frac{2 n}{n-2}>2$, e portanto podemos tomar $s>2$. Logo $\frac{1}{s}+\frac{1}{2 n /(n-2)}<\frac{1}{2}+\frac{1}{2}=1$, e portanto existe $r>0$ tal que $\frac{1}{r}+\frac{1}{s}+\frac{1}{2 n /(n-2)}=1$. Usando a desigualdade de Hölder na equação acima, obtemos

$$
\begin{aligned}
\left\|Q u_{k}-Q u\right\|_{a}^{2} & \leq\|1\|_{L^{r}}\left\|u_{k}-u\right\|_{L^{s}}\left\|Q u_{k}-Q u\right\|_{L^{2 n /(n-2)}}= \\
& =|\Omega|^{1 / r}\left\|u_{k}-u\right\|_{L^{s}}\left\|Q u_{k}-Q u\right\|_{L^{2 n /(n-2)}}
\end{aligned}
$$

Mas, pela desigualdade de Sobolev, temos $\left\|Q u_{k}-Q u\right\|_{L^{2 n /(n-2)}} \leq C_{1}\left\|Q u_{k}-Q u\right\|_{H^{1}}$ para alguma constante positiva $C_{1}$. Portanto

$$
\begin{aligned}
\left\|Q u_{k}-Q u\right\|_{a}^{2} & \leq\left(C_{1}|\Omega|^{1 / r}\right)\left\|u_{k}-u\right\|_{L^{s}}\left\|Q u_{k}-Q u\right\|_{H^{1}} \leq \\
& \leq\left(C_{1} C_{2}|\Omega|^{1 / r}\right)\left\|u_{k}-u\right\|_{L^{s}}\left\|Q u_{k}-Q u\right\|_{a}
\end{aligned}
$$

pois as normas $\|\cdot\|_{H^{1}} \mathrm{e}\|\cdot\|_{a}$ são equivalentes. 
Dessa desigualdade concluímos que

$$
\left\|Q u_{k}-Q u\right\|_{a} \leq\left(C_{1} C_{2}|\Omega|^{1 / r}\right)\left\|u_{k}-u\right\|_{L^{s}} \Longrightarrow Q u_{k} \rightarrow Q u \quad \text { em } H_{0}^{1}(\Omega)
$$

ou seja, $Q$ é compacto.

Nosso problema (4.3) pode ser reescrito como

$$
\langle u, v\rangle_{a}=\lambda\langle Q u, v\rangle_{a} \quad \forall v \in H_{0}^{1}(\Omega)
$$

$\mathrm{ou}$

$$
\lambda Q u=u \Longleftrightarrow Q u=\frac{1}{\lambda} u .
$$

Sabendo que $Q$ é compacto, o Teorema Espectral para operadores compactos e auto-adjuntos diz que os autovalores de $Q$ podem ser dispostos numa seqüência $\left(\kappa_{k}\right)_{k \geq 1}$ com a propriedade que $\left|\kappa_{k}\right| \geq\left|\kappa_{k+1}\right|$ para todo $k \geq 1$ e $\lim _{k \rightarrow \infty}\left|\kappa_{k}\right|=0$. Logo os autovalores do nosso problema podem ser dispostos numa seqüência $\left(\lambda_{k}\right)$ tal que

$$
0<\lambda_{1} \leq \lambda_{2} \leq \ldots \leq \lambda_{k} \leq \lambda_{k+1} \leq \ldots
$$

e $\lim _{k \rightarrow \infty} \lambda_{k}=+\infty$. Note que, de fato, os autovalores do nosso problema são positivos: basta tomar $v=u$ na equação (4.3). Podemos dizer ainda mais sobre o primeiro autovalor do problema:

Teorema 4.3 O primeiro autovalor $\lambda_{1}$ é dado por

$$
\inf _{u \in H_{0}^{1}(\Omega)} \frac{\int_{\Omega}|\nabla u|^{2}+\alpha \int_{\Omega} \chi_{D} u^{2}}{\int_{\Omega} u^{2}} .
$$

Além disso, $\lambda_{1}$ é simples, e sua autofunção correspondente $u_{1}$ pode ser tomada positiva em $\Omega$.

Demonstração: Para $v \in H_{0}^{1}(\Omega)$, seja

$$
R(v)=\frac{\langle v, v\rangle_{a}}{\langle v, v\rangle_{L^{2}}}
$$

em que $\langle\cdot, \cdot\rangle_{a}$ é o produto interno em $H_{0}^{1}(\Omega)$ definido na Proposição 4.1. Temos

$$
R(v) \geq \frac{\int_{\Omega}|\nabla v|^{2}}{\int_{\Omega} v^{2}} \geq C>0,
$$


para alguma constante $C$, pela desigualdade de Poincaré. Seja então

$$
\sigma=\inf _{v \in H_{0}^{1}(\Omega)} R(v)
$$

Mostraremos que $\lambda_{1}=\sigma$. Para tanto, escolhemos uma seqüência minimizante $\left(\psi_{k}\right)_{k \geq 1}$ de elementos de $H_{0}^{1}(\Omega)$ tais que $\left\|\psi_{k}\right\|_{L^{2}}=1$ e $R\left(\psi_{k}\right) \rightarrow \sigma$. A seqüência $\left(\psi_{k}\right)_{k \geq 1}$ é limitada em $H_{0}^{1}(\Omega)$ e portanto, pela compacidade da inclusão $i: H_{0}^{1}(\Omega) \rightarrow L^{2}(\Omega)$, existe uma subseqüência, que também denotaremos por $\left(\psi_{k}\right)_{k \geq 1}$, que converge em $L^{2}(\Omega)$ para uma função $\psi$ tal que $\|\psi\|_{L^{2}}=1$. Como $\|v\|_{a}^{2}=\langle v, v\rangle_{a}$ é uma forma quadrática, temos, para $k, \ell \geq 1$,

$$
\left\|\frac{\psi_{k}-\psi_{\ell}}{2}\right\|_{a}^{2}+\left\|\frac{\psi_{k}+\psi_{\ell}}{2}\right\|_{a}^{2}=\frac{1}{2}\left(\left\|\psi_{k}\right\|_{a}^{2}+\left\|\psi_{\ell}\right\|_{a}^{2}\right),
$$

$\log 0$

$$
\left\|\frac{\psi_{k}-\psi_{\ell}}{2}\right\|_{a}^{2} \leq \frac{1}{2}\left(\left\|\psi_{k}\right\|_{a}^{2}+\left\|\psi_{\ell}\right\|_{a}^{2}\right)-\sigma\left\|\frac{\psi_{k}+\psi_{\ell}}{2}\right\|_{L^{2}}^{2} \rightarrow 0 \text { quando } k, \ell \rightarrow \infty .
$$

Portanto $\left(\psi_{k}\right)_{k \geq 1}$ é uma seqüência de Cauchy em $H_{0}^{1}(\Omega)$. Assim, $\psi_{k} \rightarrow \psi$ em $H_{0}^{1}(\Omega)$ e, ainda, $\|\psi\|_{a}^{2}=\sigma$. Defina $f: \mathbb{R} \rightarrow \mathbb{R}$ por

$$
f(t)=R(\psi+t v)
$$

em que $v \in H_{0}^{1}(\Omega)$. Devemos ter $f^{\prime}(0)=0$, mas por outro lado temos

$$
\begin{aligned}
f^{\prime}(0) & =\left.\frac{d}{d t}\right|_{t=0}\left(\frac{\langle\psi+t v, \psi+t v\rangle_{a}}{\langle\psi+t v, \psi+t v\rangle_{L^{2}}}\right) \\
& =2\left(\int_{\Omega} \nabla \psi \cdot \nabla v+\alpha \int_{\Omega} \chi_{D} \psi v-\sigma \int_{\Omega} \psi v\right),
\end{aligned}
$$

o que mostra que $\sigma$ é um autovalor com autofunção correspondente $\psi$. É fácil ver que $\sigma$ é o menor autovalor, isto é, $\sigma=\lambda_{1}$, pois qualquer autovalor menor que $\sigma$ contrariaria a definição de $\sigma$ dada por (4.9).

Mostremos agora que podemos tomar $u_{1}=\psi$ positiva em $\Omega$. A função $u_{1}$ é solução de

$$
\left\langle u_{1}, v\right\rangle_{a}=\lambda_{1} \int_{\Omega} u_{1} v \quad \forall v \in H_{0}^{1}(\Omega) .
$$


Suponha que $u_{1}$ mude de sinal em $\Omega$, e sejam $u_{1}^{+}=\operatorname{máx}\left(u_{1}, 0\right)$ e $u_{1}^{-}=\operatorname{mín}\left(u_{1}, 0\right)$. Sabemos que $u_{1}^{+}$ e $u_{1}^{-}$pertencem a $H_{0}^{1}(\Omega)$ (veja, por exemplo, [24, Corolário 2.1.8]). Temos

$$
\begin{aligned}
\int_{\Omega} u_{1}^{2} & =\int_{\Omega}\left(u_{1}^{+}\right)^{2}+\int_{\Omega}\left(u_{1}^{-}\right)^{2}=\alpha_{1}+\alpha_{2} \\
\left\langle u_{1}, u_{1}\right\rangle_{a} & =\left\langle u_{1}^{+}, u_{1}^{+}\right\rangle_{a}+\left\langle u_{1}^{-}, u_{1}^{-}\right\rangle_{a}=\beta_{1}+\beta_{2},
\end{aligned}
$$

$\log 0$

$$
\frac{1}{\lambda_{1}}=\frac{\int_{\Omega} u_{1}^{2}}{\left\langle u_{1}, u_{1}\right\rangle_{a}}=\frac{\alpha_{1}+\alpha_{2}}{\beta_{1}+\beta_{2}} \leq \operatorname{máx}\left\{\frac{\alpha_{1}}{\beta_{1}}, \frac{\alpha_{2}}{\beta_{2}}\right\},
$$

com igualdade se, e somente se, $\frac{\alpha_{1}}{\beta_{1}}=\frac{\alpha_{2}}{\beta_{2}}$, de onde concluímos que $u_{1}^{+}$e $u_{1}^{-}$são também autofunções correspondentes a $\lambda_{1}$. De acordo com [22, corolário 8.1], $u_{1}^{+}>0$ quase sempre e $u_{1}^{-}<0$ quase sempre, o que é impossível. Logo $u_{1}$ não muda de sinal em $\Omega$. O fato que $u_{1}$ pode ser tomada positiva segue deste mesmo resultado em [22].

Provemos agora que a multiplicidade geométrica de $\lambda_{1}$ é 1 . Sejam $f_{1}$ e $f_{2}$ autofunções correspondentes a $\lambda_{1}$. Sabemos que para cada $t \in \mathbb{R}$ a autofunção $f_{1}+t f_{2}$ tem um sinal definido. Desse modo, sejam

$$
A=\left\{t \in \mathbb{R}: f_{1}+t f_{2} \geq 0\right\} \quad \text { e } \quad B=\left\{t \in \mathbb{R}: f_{1}+t f_{2} \leq 0\right\} .
$$

Os conjuntos $A$ e $B$ são não vazios, fechados e $A \cup B=\mathbb{R}$. Conseqüentemente existe $\bar{t} \in A \cap B \Rightarrow$ $f_{1}+\bar{t} f_{2}=0$, o que significa que $f_{1}$ e $f_{2}$ são linearmente dependentes.

Finalmente mostremos que a multiplicidade algébrica de $\lambda_{1}$ também é 1 , isto é, que $\operatorname{Ker}(I-$ $\left.\lambda_{1} Q\right)^{2}=\operatorname{Ker}\left(I-\lambda_{1} Q\right)$. Seja $u \in \operatorname{Ker}\left(I-\lambda_{1} Q\right)^{2}$. Então $u-\lambda_{1} Q u=t u_{1}$, em que $u_{1}$ é a autofunção positiva de $\lambda_{1}$ e $t$ é algum número real. Tomando o produto interno com $u_{1}$, obtemos

$$
t\left\langle u_{1}, u_{1}\right\rangle_{a}=\left\langle u-\lambda_{1} Q u, u_{1}\right\rangle_{a}=\left\langle u, u_{1}-\lambda_{1} Q u_{1}\right\rangle_{a}=0,
$$

o que implica $t=0$, logo $u \in \operatorname{Ker}\left(I-\lambda_{1} Q\right)$, e a demonstração está completa.

Observação: Um raciocínio análogo mostra que o primeiro autovalor do problema $(*)_{\Omega, \rho}$ é dado por

$$
\inf _{u \in H_{0}^{1}(\Omega)} \frac{\int_{\Omega}|\nabla u|^{2}}{\int_{\Omega} \rho u^{2}} .
$$


Seja $T$ o operador $-\Delta+\alpha \chi_{D}$, isto é,

$$
\begin{aligned}
T: H_{0}^{1}(\Omega) \cap H^{2}(\Omega) \subset L^{2}(\Omega) & \rightarrow L^{2}(\Omega) \\
u & \mapsto-\Delta u+\alpha \chi_{D} u .
\end{aligned}
$$

Já mostramos que os autovalores de $T$ formam uma seqüência positiva que tende a $+\infty$. Nosso próximo objetivo é mostrar que $T$ é um isomorfismo. Para tanto, faremos uso dos resultados do Capítulo 2.

Proposição 4.2 O operador $T+\Delta: H_{0}^{1}(\Omega) \cap H^{2}(\Omega) \rightarrow L^{2}(\Omega)$ é compacto.

Demonstração: Consideremos o operador

$$
\begin{aligned}
X: H_{0}^{1}(\Omega) & \rightarrow L^{2}(\Omega) \\
u & \mapsto\left(\alpha \chi_{D}\right) u
\end{aligned}
$$

A inclusão $i: H_{0}^{1}(\Omega) \rightarrow L^{2}(\Omega)$ é compacta, o operador $X_{1}: L^{2}(\Omega) \rightarrow L^{2}(\Omega)$ dado por $X_{1}(u)=\left(\alpha \chi_{D}\right) u$ é contínuo e $X=X_{1} \circ i$. Pelo Lema $2.3, X$ é compacto. Se $j: H_{0}^{1}(\Omega) \cap H^{2}(\Omega) \rightarrow H_{0}^{1}(\Omega)$ é a inclusão, então $X \circ j=T+\Delta$ é compacto.

Proposição 4.3 $T: H_{0}^{1}(\Omega) \cap H^{2}(\Omega) \rightarrow L^{2}(\Omega)$ é um isomorfismo.

Demonstração: Mostremos primeiro que $T$ é injetor: seja $u \in \operatorname{Ker}(T) \Longleftrightarrow T u=0$. Então

$$
0=\int_{\Omega} T u \cdot u=\int_{\Omega}|\nabla u|^{2}+\alpha \chi_{D}|u|^{2} \Longrightarrow u=0 .
$$

Para mostrar que $T$ é sobrejetor, basta mostrar que $T$ é Fredholm e $i_{F}(T)=0$, pois já temos $\operatorname{dim}(\operatorname{Ker} T)=0$. Mas isto é simples: o operador $-\Delta: H_{0}^{1}(\Omega) \cap H^{2}(\Omega) \rightarrow L^{2}(\Omega)$ é isomorfismo [12, Teorema 9.15], logo é um operador de Fredholm e $i_{F}(-\Delta)=0$. Portanto, pela Proposição 2.5, o operador $T=(T+\Delta)+(-\Delta)$ é Fredholm e $i_{F}(T)=i_{F}(-\Delta)=0$.

Nosso próximo passo consiste em mostrar que $T^{-1}: L^{2}(\Omega) \rightarrow L^{2}(\Omega)$ é compacto. 
Proposição 4.4 $T^{-1}: L^{2}(\Omega) \rightarrow H_{0}^{1}(\Omega) \cap H^{2}(\Omega)$ é contínuo.

Demonstração: Dada $f \in L^{2}(\Omega)$, seja $u=T^{-1} f \Longleftrightarrow-\Delta u+\alpha \chi_{D} u=f$. Multiplicando essa igualdade por $u$ e integrando, temos

$$
\left(\|u\|_{H^{1}}^{*}\right)^{2}=\|\nabla u\|_{L^{2}}^{2} \leq \int_{\Omega}|\nabla u|^{2}+\alpha \int_{D}|u|^{2}=\int_{\Omega} f u \leq\|f\|_{L^{2}}\|u\|_{L^{2}}
$$

Daí:

$$
\begin{gathered}
\left(\|u\|_{H^{1}}^{*}\right)^{2}=\|\nabla u\|_{L^{2}}^{2} \leq\|f\|_{L^{2}}\|u\|_{L^{2}} \Longrightarrow \\
\|u\|_{H^{1}}^{*} \leq\|f\|_{L^{2}} \frac{\|u\|_{L^{2}}}{\|u\|_{H^{1}}^{*}} \leq C\|f\|_{L^{2}},
\end{gathered}
$$

pois $\|u\|_{L^{2}} /\|u\|_{H^{1}}^{*} \leq C$, para algum $C>0$.

Pela Proposição 4.4 e pelo Lema 2.3, o operador $T^{-1}: L^{2}(\Omega) \rightarrow L^{2}(\Omega)$ é compacto. Claramente o operador $T^{-1}$ é auto-adjunto. Poderíamos então aplicar novamente o teorema Espectral para descobrir que a seqüência de autovalores de $T$ é formada por elementos positivos e que tendem a $+\infty$. 


\section{Capítulo 5}

\section{Fórmulas de variação para o primeiro autovalor}

Já obtivemos os resultados principais a respeito do operador $T=-\Delta+\alpha \chi_{D}$, que aparece no problema de autovalores $(*)_{\Omega, \alpha, D}$. Agora, estudaremos o problema variacional para o problema das membranas compostas: dado um conjunto $D$, estudaremos o que ocorre com o primeiro autovalor $\lambda_{\Omega}(\alpha, D)$ quando variamos $D$.

Antes de mais nada vamos fixar a notação: seja $\Gamma=\partial D \cap \Omega$, isto é, $\Gamma$ é a parte da fronteira de $D$ interna a $\Omega$, e seja $\mu$ a normal exterior a $\Omega$. Em seguida, vamos considerar uma variação de $\Omega$, isto é, considere uma função de classe $C^{2}$

$$
\varphi:(-\varepsilon, \varepsilon) \times \bar{\Omega} \rightarrow \bar{\Omega}
$$

tal que para cada $t \in(-\varepsilon, \varepsilon), \varphi_{t}=\varphi(t, \cdot)$ é um difeomorfismo de $\bar{\Omega}$. Vamos supor que $\varphi_{t}$ é um subgrupo a 1 parâmetro, isto é,

$$
\begin{gathered}
\varphi_{0}=\operatorname{Id}_{\bar{\Omega}}, \\
t, s, t+s \in(-\varepsilon, \varepsilon) \Longrightarrow \varphi_{t} \circ \varphi_{s}=\varphi_{t+s} .
\end{gathered}
$$

Em particular, temos $\varphi_{t}^{-1}=\varphi_{-t}$, e note que a restrição de $\varphi$ a $D$ também é uma variação. O campo $V$ é definido por

$$
V(x)=\left.\frac{d}{d t}\right|_{t=0} \varphi_{t}(x)
$$


Sejam ainda $D_{t}=\varphi_{t}(D), \lambda(t)=\lambda_{\Omega}\left(\alpha, D_{t}\right)$.

\subsection{Primeiro método: primeira variação}

Seja $\tilde{u}_{t}$ a autofunção positiva e normalizada em $L^{2}(\Omega)$ que corresponde a $\lambda(t)$. Então $\tilde{u}_{t}$ satisfaz

$$
\left\{\begin{array}{l}
-\Delta \tilde{u}_{t}+\alpha \chi_{D_{t}} \tilde{u}_{t}=\lambda(t) \tilde{u}_{t} \quad \text { em } \Omega_{t} \\
\left.\tilde{u}_{t}\right|_{\partial \Omega_{t}}=0
\end{array}\right.
$$

Calculemos a primeira variação para $\lambda$. A técnica utilizada é baseada no artigo [20]. Seja $A_{t}: C^{2}(\Omega) \rightarrow C^{0}(\Omega)$ o operador $A_{t}=-\varphi_{t}^{*} \Delta \varphi_{-t}^{*}$, isto é, $A_{t}(f)=-\Delta\left(f \circ \varphi_{-t}\right) \circ \varphi_{t}$. Sendo $u_{t}=\tilde{u}_{t} \circ \varphi_{t}$, temos que $u_{t}$ está definida em $\Omega, \log 0$

$$
A_{t} u_{t}+\alpha \chi_{D} u_{t}=\lambda(t) u_{t},\left.\quad u_{t}\right|_{\partial \Omega}=0
$$

Mostraremos que $\left.\frac{d}{d t}\right|_{t=0} A_{t}(f)=[\Delta, V](f)=\Delta(V(f))-V(\Delta f)$ para $f \in C^{2}(\Omega)$. Para tanto, sejam $B_{t}=-A_{t}$ e $g_{t}=\Delta\left(f \circ \varphi_{-t}\right)$. Temos então $B_{t}=g_{t} \circ \varphi_{t}$. Para as contas que realizaremos a seguir, será útil usarmos a seguinte notação: dada uma função qualquer $\zeta_{t}(x)$, com $t \in(-\varepsilon, \varepsilon)$ e $x \in \mathbb{R}^{n}$, escreveremos $\zeta(t, x)$ no lugar de $\zeta_{t}(x)$. O único efeito da notação $\zeta(t, x)$ é explicitar a composição de funções. Temos então

$$
\begin{gathered}
B_{t}(f)(x)=\left(g_{t} \circ \varphi_{t}\right)(x)=g(t, \varphi(t, x)) \Longrightarrow \\
\frac{d}{d t}\left(B_{t}(f)(x)\right)=\frac{\partial g}{\partial t}(t, \varphi(t, x)) \cdot \frac{\partial t}{\partial t}+\sum_{i=1}^{n} \frac{\partial g}{\partial x_{i}}(t, \varphi(t, x)) \frac{\partial \varphi_{i}}{\partial t}(t, x) \Longrightarrow \\
\left.\frac{d}{d t}\right|_{t=0}\left(B_{t}(f)(x)\right)=\frac{\partial g}{\partial t}(0, x)+\left\langle\nabla g_{0}(x), V(x)\right\rangle= \\
=\frac{\partial g}{\partial t}(0, x)+\langle\nabla(\Delta f)(x), V(x)\rangle=\frac{\partial g}{\partial t}(0, x)+V(\Delta f)(x) .
\end{gathered}
$$

A tarefa que nos resta, portanto, é calcular $\frac{\partial g}{\partial t}(0, x)$. Observando que $g(t, x)=\Delta\left(f \circ \varphi_{-t}\right)(x)=$ 
$\Delta(f(\varphi(-t, x)))$,

$$
\begin{aligned}
\frac{\partial g}{\partial t}(t, x) & =\Delta\left(\frac{\partial}{\partial t} f(\varphi(-t, x))\right)=\Delta\left(\frac{\partial}{\partial t} f\left(\varphi_{1}(-t, x), \ldots, \varphi_{n}(-t, x)\right)\right)= \\
& =\Delta\left(\sum_{i=1}^{n} \frac{\partial f}{\partial x_{i}}(\varphi(-t, x)) \cdot \frac{\partial \varphi_{i}}{\partial t}(-t, x) \cdot(-1)\right)= \\
& =-\Delta\left(\sum_{i=1}^{n} \frac{\partial f}{\partial x_{i}}(\varphi(-t, x)) \cdot \frac{\partial \varphi_{i}}{\partial t}(-t, x)\right),
\end{aligned}
$$

e esta fórmula para $t=0$ nos dá

$$
\begin{aligned}
\frac{\partial g}{\partial t}(0, x) & =-\Delta\left(\sum_{i=1}^{n} \frac{\partial f}{\partial x_{i}}(\varphi(0, x)) \cdot \frac{\partial \varphi_{i}}{\partial t}(0, x)\right)= \\
& =-\Delta\left(\sum_{i=1}^{n} \frac{\partial f}{\partial x_{i}}(x) \cdot \frac{\partial \varphi_{i}}{\partial t}(0, x)\right)= \\
& =-\Delta(\langle\nabla f(x), V(x)\rangle)=-\Delta(V(f))(x) .
\end{aligned}
$$

$\left.\log \frac{d}{d t}\right|_{t=0} B_{t}(f)=-\Delta(V(f))+\left.V(\Delta f) \Longleftrightarrow \frac{d}{d t}\right|_{t=0} A_{t}(f)=\Delta(V(f))-V(\Delta f)$

Um cálculo semelhante nos mostra que

$$
\begin{aligned}
\left.\frac{\partial}{\partial t}\right|_{t=0}(f(t, \varphi(-t, x)))= & {\left[\frac{\partial f}{\partial t}(t, \varphi(-t, x)) \cdot 1+\right.} \\
& \left.\quad+\sum_{i=1}^{n} \frac{\partial f}{\partial x_{i}}(t, \varphi(-t, x)) \cdot \frac{\partial \varphi_{i}}{\partial t}(-t, x) \cdot(-1)\right]_{t=0} \\
= & {\left[\frac{\partial f}{\partial t}(t, \varphi(-t, x))-\sum_{i=1}^{n} \frac{\partial f}{\partial x_{i}}(t, \varphi(-t, x)) \cdot \frac{\partial \varphi_{i}}{\partial t}(-t, x)\right]_{t=0} } \\
= & \frac{\partial f}{\partial t}(0, x)-\sum_{i=1}^{n} \frac{\partial f}{\partial x_{i}}(0, x) \cdot \frac{\partial \varphi_{i}}{\partial t}(0, x) \\
= & \frac{\partial f}{\partial t}(0, x)-V(f)(x)
\end{aligned}
$$


o que implica, para $\zeta:(-\varepsilon, \varepsilon) \times \Omega \rightarrow \mathbb{R}$,

$$
\left.\frac{d}{d t}\right|_{t=0} A_{t} \zeta_{t}=A^{\prime} \zeta-\Delta \zeta^{\prime}
$$

Na equação acima, o símbolo ' representa a derivada em relação a $t$ calculada para $t=0$.

Derivando a equação (5.1) e calculando para $t=0$, obtemos, usando (5.2),

$$
\begin{aligned}
& A^{\prime} u-\Delta u^{\prime}+\alpha \chi_{D} u^{\prime}=\lambda^{\prime}(0) u+\lambda u^{\prime} \Longleftrightarrow \\
& \Longleftrightarrow \Delta u^{\prime}-\alpha \chi_{D} u^{\prime}+\lambda u^{\prime}=A^{\prime} u-\lambda^{\prime}(0) u,
\end{aligned}
$$

em que $u=u_{0}, u^{\prime}=u_{0}^{\prime}$ e $\lambda=\lambda(0)$. Logo $A^{\prime} u-\lambda^{\prime}(0) u \in \operatorname{Im}\left(\Delta-\alpha \chi_{D}+\lambda\right)=\operatorname{Ker}\left(\Delta-\alpha \chi_{D}+\lambda\right)^{\perp}=[u]^{\perp}$ (o operador $\Delta-\alpha \chi_{D}+\lambda$ tem imagem fechada, pois é Fredholm. A verificação desse fato é análoga à demonstração de que $-\Delta+\alpha \chi_{D}$ é Fredholm). Mas

$$
\begin{gathered}
\left\langle A^{\prime} u-\lambda^{\prime}(0) u, u\right\rangle=0 \Longleftrightarrow \int_{\Omega}\left(A^{\prime} u-\lambda^{\prime}(0) u\right) u=0 \Longleftrightarrow \\
\Longleftrightarrow \int_{\Omega} u A^{\prime} u=\lambda^{\prime}(0) \int_{\Omega} u^{2}=\lambda^{\prime}(0) \Longleftrightarrow \lambda^{\prime}(0)=\int_{\Omega} u[\Delta(V(u))-V(\Delta u)] .
\end{gathered}
$$

Observando que $\Delta u=\alpha \chi_{D} u-\lambda u$, temos

$$
\begin{aligned}
\lambda^{\prime}(0) & =\int_{\Omega} u \Delta(V(u))-u V(\Delta u) \\
& =\int_{\Omega} u \Delta(V(u))-u V\left(\alpha \chi_{D} u-\lambda u\right) \\
& =\int_{\Omega} u \Delta(V(u))+\lambda u V(u)-u V\left(\alpha \chi_{D} u\right) \\
& =\int_{\Omega} u \Delta(V(u))+\left(\alpha \chi_{D} u-\Delta u\right) V(u)-u V\left(\alpha \chi_{D} u\right) \\
& =\int_{\Omega}[u \Delta(V(u))-V(u) \Delta u]+\alpha \int_{\Omega}\left(\chi_{D} u V(u)-u V\left(\chi_{D} u\right)\right) .
\end{aligned}
$$

Teorema 5.1 (Primeira variação) Se $\varphi$ é uma variação que deixa fixo cada ponto de $\partial \Omega$, então

$$
\lambda^{\prime}(0)=\alpha \int_{\Gamma} u^{2}\langle V, \nu\rangle d S
$$


em que $\nu$ é a normal exterior a $\Gamma$, relativa ao conjunto $D$.

Demonstração: Observando a última linha da equação (5.3), podemos aplicar a segunda identidade de Green no primeiro termo para obter

$$
\int_{\Omega}[u \Delta(V(u))-V(u) \Delta u]=\int_{\partial \Omega}(u\langle\nabla V(u), \mu\rangle-V(u)\langle\nabla u, \mu\rangle) d S=0,
$$

pois $\left.u\right|_{\partial \Omega}=0$ e $V=0$ em $\partial \Omega$ se $\varphi$ fixa cada ponto de $\partial \Omega$. Temos então

$$
\begin{aligned}
\lambda^{\prime}(0) & =\alpha \int_{\Omega}\left(\chi_{D} u V(u)-u V\left(\chi_{D} u\right)\right) \\
& =-\alpha \int_{\Omega} u^{2} V\left(\chi_{D}\right) \\
& =\alpha \int_{\Omega} \chi_{D} \operatorname{div}\left(u^{2} V\right)-\alpha \int_{\Omega} \operatorname{div}\left(\chi_{D} u^{2} V\right) \\
& =\alpha \int_{D} \operatorname{div}\left(u^{2} V\right),
\end{aligned}
$$

já que o segundo termo na penúltima linha é zero pelo teorema da Divergência de Gauss. Terminamos a demonstração aplicando novamente o teorema da Divergência:

$$
\alpha \int_{D} \operatorname{div}\left(u^{2} V\right)=\alpha \int_{\Gamma} u^{2}\langle V, \nu\rangle d S+\alpha \int_{\partial \Omega} u^{2}\langle V, n\rangle d S=\alpha \int_{\Gamma} u^{2}\langle V, \nu\rangle d S
$$

pois $u=0$ em $\partial \Omega$.

Note que a fórmula para a primeira variação vale para qualquer $\varphi$ que fixe a fronteira de $\Omega$, preservando ou não a medida de $D$.

Observação: Uma dificuldade bastante aparente que estamos ignorando até o momento (em especial no último teorema) é a seguinte: a função $\chi_{D}$ não é contínua, o que nos diz imediatamente que a função $u$ não pertence a $C^{2}(\Omega)$. Note que o operador $A_{t}$ que introduzimos foi definido em $C^{2}(\Omega)$, pois $\Delta$ é um operador de segunda ordem. Uma expressão como $V\left(\chi_{D}\right)$, a rigor, não faz nenhum sentido. Porém, os nossos cálculos podem ser justificados. A função $\chi_{D}$ é de variação limitada, e para tais funções podemos interpretar o símbolo $V\left(\chi_{D}\right)$. Além disso, o Teorema da Divergência de Gauss vale para tais funções. Uma referência para a teoria de funções de variação 
limitada pode ser encontrado em [13].

\subsection{Segundo método: primeira e segunda variações}

A mesma técnica usada no cálculo da primeira variação pode também ser usada para calcular a segunda variação para $\lambda$, mas existe outro método mais direto para obtermos nossos resultados. Consideraremos que a variação $\varphi$ deixa fixo cada ponto de $\partial \Omega$, isto é $\varphi_{t}$ deixa fixo cada ponto de $\partial \Omega$ para todo $t \in(-\varepsilon, \varepsilon)$. Ou então podemos pensar que $\varphi_{t}$ tem suporte compacto em $\Omega$ para todo $t \in(-\varepsilon, \varepsilon)$. Representaremos novamente por $\lambda(t)$ o primeiro autovalor de

$$
\mid \begin{aligned}
& -\Delta u+\alpha \chi_{D_{t}} u=\lambda u \\
& u=0 \quad \text { em } \partial \Omega
\end{aligned},
$$

e $u_{t}$ representará a primeira autofunção correspondente a $\lambda(t)$, normalizada em $L^{2}(\Omega)$. Também definimos $u=u_{0}$ e $\lambda=\lambda(0)$. Temos

$$
\mid \begin{aligned}
& -u_{t} \Delta u+\alpha \chi_{D} u u_{t}=\lambda u u_{t} \\
& -u \Delta u_{t}+\alpha \chi_{D_{t}} u u_{t}=\lambda(t) u u_{t}
\end{aligned}
$$

Subtraindo as duas equações e integrando sobre $\Omega$, obtemos

$$
-\int_{\Omega}\left(u \Delta u_{t}-u_{t} \Delta u\right)+\alpha \int_{\Omega}\left(\chi_{D_{t}}-\chi_{D}\right) u u_{t}=(\lambda(t)-\lambda) \int_{\Omega} u u_{t} .
$$

A primeira integral do lado esquerdo é zero, pois $u \Delta u_{t}-u_{t} \Delta u=\operatorname{div}\left(u \nabla u_{t}-u_{t} \nabla u\right)$, e pelo Teorema da Divergência temos

$$
\int_{\Omega} \operatorname{div}\left(u \nabla u_{t}-u_{t} \nabla u\right)=\int_{\partial \Omega}\left\langle u \nabla u_{t}-u_{t} \nabla u, n\right\rangle=0
$$

pois $u=u_{t}=0$ em $\partial \Omega$. Temos então

$$
\alpha \int_{\Omega}\left(\chi_{D_{t}}-\chi_{D}\right) u u_{t}=(\lambda(t)-\lambda) \int_{\Omega} u u_{t}
$$

Nossa idéia é derivar a equação (5.4) duas vezes relação a $t$. Porém a função característica $\chi_{D_{t}}$ não é derivável. Mesmo assim, ignorando por um momento esta dificuldade, derivamos em relação a 
$t$ e obtemos

$$
\alpha \int_{\Omega} \chi_{D_{t}}^{\prime} u u_{t}+\alpha \int_{\Omega}\left(\chi_{D_{t}}-\chi_{D}\right) u u_{t}^{\prime}=\lambda^{\prime}(t) \int_{\Omega} u u_{t}+(\lambda(t)-\lambda) \int_{\Omega} u u_{t}^{\prime},
$$

e se derivarmos uma segunda vez em relação a $t$ obtemos

$$
\begin{aligned}
& \alpha\left(\int_{\Omega} \chi_{D_{t}}^{\prime \prime} u u_{t}+2 \int_{\Omega} \chi_{D_{t}}^{\prime} u u_{t}^{\prime}+\int_{\Omega} \chi_{D_{t}} u u_{t}^{\prime \prime}-\int_{\Omega} \chi_{D} u u_{t}^{\prime \prime}\right)= \\
& \quad=\lambda^{\prime \prime}(t) \int_{\Omega} u u_{t}+2 \lambda^{\prime}(t) \int_{\Omega} u u_{t}^{\prime}+\lambda(t) \int_{\Omega} u u_{t}^{\prime \prime}-\lambda \int_{\Omega} u u_{t}^{\prime \prime} .
\end{aligned}
$$

Calculando para $t=0$, e observando que $\int_{\Omega} u u_{0}^{\prime}=0$ (pois $u_{t}$ tem norma 1 em $L^{2}(\Omega)$ para todo $t$ ), as equações (5.5) e (5.6) se transformam em

$$
\lambda^{\prime}(0)=\alpha \int_{\Omega} \chi_{D_{0}}^{\prime} u^{2}
$$

$\mathrm{e}$

$$
\lambda^{\prime \prime}(0)=\alpha\left(\int_{\Omega} \chi_{D_{0}}^{\prime \prime} u^{2}+2 \int_{\Omega} \chi_{D_{0}}^{\prime} u u_{0}^{\prime}\right)
$$

Para entendermos o significado de $\chi_{D_{0}}^{\prime}$ e $\chi_{D_{0}}^{\prime \prime}$, observe que $\chi_{D_{t}}=\chi_{D} \circ \varphi_{-t}$. De fato,

$$
x \in D_{t} \Longleftrightarrow x \in \varphi_{t}(D) \Longleftrightarrow \varphi_{t}^{-1}(x) \in D \Longleftrightarrow \varphi_{-t}(x) \in D .
$$

A função $\chi_{D} \circ \varphi_{-t}$ ainda não pode ser derivada, mas como a função $\chi_{D} \in L^{2}(\Omega)$, pois $\Omega$ é limitado, podemos aproximá-la por funções de classe $C^{\infty}$ e então derivar.

Assim, podemos tomar funções $h_{\delta} \in C_{0}^{\infty}(\Omega)$ tais que, quando $\delta \rightarrow 0, h_{\delta} \rightarrow \chi_{D}$ em $L^{2}(\Omega)$ (pois $C_{0}^{\infty}(\Omega)$ é denso em $L^{2}(\Omega)$ ). A função $h_{\delta} \circ \varphi_{-t}$ pode ser derivada em relação a $t$, e

$$
\frac{d}{d t}\left(h_{\delta} \circ \varphi_{-t}\right)(x)=-\sum_{i=1}^{n} \frac{\partial h_{\delta}}{\partial x_{i}}(\varphi(-t, x)) \cdot \frac{\partial \varphi_{i}}{\partial t}(-t, x),
$$

que calculada para $t=0$ nos fornece

$$
\left.\frac{d}{d t}\left(h_{\delta} \circ \varphi_{-t}\right)(x)\right|_{t=0}=-\left\langle\nabla h_{\delta}(x), V(x)\right\rangle=-V\left(h_{\delta}(x)\right) .
$$


Assim,

$$
\begin{aligned}
\left.\alpha \int_{\Omega}\left(h_{\delta} \circ \varphi_{-t}\right)^{\prime}\right|_{t=0} u^{2} & =-\alpha \int_{\Omega}\left\langle\nabla h_{\delta}, u^{2} V\right\rangle \\
& =-\alpha \int_{\Omega}\left(\operatorname{div}\left(h_{\delta} u^{2} V\right)-h_{\delta} \operatorname{div}\left(u^{2} V\right)\right) \\
& =-\alpha \int_{\Omega} \operatorname{div}\left(h_{\delta} u^{2} V\right)+\alpha \int_{\Omega} h_{\delta} \operatorname{div}\left(u^{2} V\right) \\
& =-\alpha \int_{\partial \Omega}\left\langle h_{\delta} u^{2} V, n\right\rangle d S+\alpha \int_{\Omega} h_{\delta} \operatorname{div}\left(u^{2} V\right) \\
& =\alpha \int_{\Omega} h_{\delta} \operatorname{div}\left(u^{2} V\right) .
\end{aligned}
$$

Quando $\delta \rightarrow 0, h_{\delta} \stackrel{L^{2}(\Omega)}{\longrightarrow} \chi_{D}$. Além disso, como $|\Omega|<\infty, \operatorname{div}\left(u^{2} V\right) \in L^{2}(\Omega)$ e portanto

$$
\left\langle h_{\delta}, \operatorname{div}\left(u^{2} V\right)\right\rangle_{L^{2}} \rightarrow\left\langle\chi_{D}, \operatorname{div}\left(u^{2} V\right)\right\rangle_{L^{2}}
$$

isto é,

$$
\alpha \int_{\Omega} h_{\delta} \operatorname{div}\left(u^{2} V\right) \rightarrow \alpha \int_{D} \operatorname{div}\left(u^{2} V\right)=\alpha \int_{\Gamma} u^{2}\langle V, \nu\rangle d S
$$

Logo, usando (5.7), a fórmula para a primeira variação é

$$
\lambda^{\prime}(0)=\alpha \int_{\Gamma} u^{2}\langle V, \nu\rangle d S
$$

como havíamos obtido anteriormente (não demonstramos que, quando $\delta \rightarrow 0$, o limite que obtemos é realmente $\lambda^{\prime}(0)$. Estamos assumindo inclusive que $\lambda(t)$ é derivável em zero).

Calculemos agora a segunda variação. Repetindo o raciocínio que fizemos acima para o cálculo da primeira variação, quando $\delta \rightarrow 0$,

$$
\left.\alpha \int_{\Omega}\left(h_{\delta} \circ \varphi_{-t}\right)^{\prime}\right|_{t=0} u u_{0}^{\prime} \rightarrow \alpha \int_{\Gamma} u u_{0}^{\prime}\langle V, \nu\rangle d S .
$$

Derivando $\left(h_{\delta} \circ \varphi_{-t}\right)$ duas vezes em relação a $t$ e calculando para $t=0$, obtemos

$$
\left.\frac{d^{2}}{d t^{2}}\left(h_{\delta} \circ \varphi_{-t}\right)(x)\right|_{t=0}=V^{2}\left(h_{\delta}(x)\right) .
$$


A igualdade anterior não é verdadeira em geral, mas vale quando $\varphi_{t}$ é um subgrupo a um parâmetro. De fato, temos

$$
\begin{aligned}
\left.\frac{d^{2}}{d t^{2}}\left(h_{\delta} \circ \varphi_{-t}\right)(x)\right|_{t=0} & =\sum_{i, j=1}^{n} \frac{\partial^{2} h_{\delta}}{\partial x_{j} \partial x_{i}}(x) \frac{\partial \varphi_{i}}{\partial t}(0, x) \frac{\partial \varphi_{j}}{\partial t}(0, x)+ \\
& +\sum_{i=1}^{n} \frac{\partial h_{\delta}}{\partial x_{i}}(x) \frac{\partial^{2} \varphi_{i}}{\partial t^{2}}(0, x)
\end{aligned}
$$

e

$$
\begin{aligned}
V^{2}\left(h_{\delta}\right) & =\sum_{i, j=1}^{n} \frac{\partial^{2} h_{\delta}}{\partial x_{j} \partial x_{i}}(x) \frac{\partial \varphi_{i}}{\partial t}(0, x) \frac{\partial \varphi_{j}}{\partial t}(0, x)+ \\
& +\sum_{i, j=1}^{n} \frac{\partial h_{\delta}}{\partial x_{i}}(x) \frac{\partial^{2} \varphi_{i}}{\partial x_{j} \partial t}(0, x) \frac{\partial \varphi_{j}}{\partial t}(0, x)
\end{aligned}
$$

o que mostra que não vale $\left.\frac{d^{2}}{d t^{2}}\left(h_{\delta} \circ \varphi_{-t}\right)(x)\right|_{t=0}=V^{2}\left(h_{\delta}\right)$ em geral. Porém, se $\varphi_{t}$ é um subgrupo a um parâmetro,

$$
\varphi_{a+b}=\varphi_{a} \circ \varphi_{b} \Longrightarrow \varphi_{i}(a+b, x)=\varphi_{i}(a, \varphi(b, x)) .
$$

Derivando essa identidade em relação a $a$, ficamos com

$$
\frac{\partial \varphi_{i}}{\partial t}(a+b, x)=\frac{\partial \varphi_{i}}{\partial t}(a, \varphi(b, x))
$$

e se derivarmos essa última igualdade em relação a $b$, finalmente obtemos

$$
\frac{\partial^{2} \varphi_{i}}{\partial t^{2}}(a+b, x)=\sum_{j=1}^{n} \frac{\partial^{2} \varphi_{i}}{\partial x_{j} \partial t}(a, \varphi(b, x)) \frac{\partial \varphi_{j}}{\partial t}(b, x) .
$$

Tomando $a=b=0$ nesta última igualdade, obtemos

$$
\frac{\partial^{2} \varphi_{i}}{\partial t^{2}}(0, x)=\sum_{j=1}^{n} \frac{\partial^{2} \varphi_{i}}{\partial x_{j} \partial t}(0, x) \frac{\partial \varphi_{j}}{\partial t}(0, x),
$$

o que implica na igualdade entre $\left.\frac{d^{2}}{d t^{2}}\left(h_{\delta} \circ \varphi_{-t}\right)(x)\right|_{t=0}$ e $V^{2}\left(h_{\delta}\right)$. 
Retornando ao cálculo da segunda variação, definindo $\ell_{\delta}=V\left(h_{\delta}\right)$, temos

$$
\begin{aligned}
\left.\alpha \int_{\Omega}\left(h_{\delta} \circ \varphi_{-t}\right)^{\prime \prime}\right|_{t=0} u^{2} & =\alpha \int_{\Omega} V^{2}\left(h_{\delta}\right) u^{2} \\
& =\alpha \int_{\Omega} V\left(\ell_{\delta}\right) u^{2} \\
& =\alpha \int_{\Omega}\left\langle\nabla \ell_{\delta}, u^{2} V\right\rangle \\
& =\alpha \int_{\Omega}\left(\operatorname{div}\left(\ell_{\delta} u^{2} V\right)-\ell_{\delta} \operatorname{div}\left(u^{2} V\right)\right) \\
& =\alpha \int_{\partial \Omega} \ell_{\delta} u^{2}\langle V, \mu\rangle d S-\alpha \int_{\Omega} \ell_{\delta} \operatorname{div}\left(u^{2} V\right)= \\
& =-\alpha \int_{\Omega}\left\langle\nabla h_{\delta}, V\right\rangle \operatorname{div}\left(u^{2} V\right) \\
& =-\alpha \int_{\Omega}\left\langle\nabla h_{\delta}, \operatorname{div}\left(u^{2} V\right) V\right\rangle \\
& =-\alpha \int_{\Omega}\left(\operatorname{div}\left(h_{\delta} \operatorname{div}\left(u^{2} V\right) V\right)-h_{\delta} \operatorname{div}\left(\operatorname{div}\left(u^{2} V\right) V\right)\right) \\
& =-\alpha \int_{\partial \Omega} h_{\delta} \operatorname{div}\left(u^{2} V\right)\langle V, \mu\rangle d S+\alpha \int_{\Omega} h_{\delta} \operatorname{div}\left(\operatorname{div}\left(u^{2} V\right) V\right) .
\end{aligned}
$$

Porém, $\operatorname{div}\left(u^{2} V\right)=2 u\langle\nabla u, V\rangle+u^{2} \operatorname{div} V$, que é igual a zero em $\partial \Omega$. Assim, quando $\delta \rightarrow 0$, $\left\langle h_{\delta}, \operatorname{div}\left(\operatorname{div}\left(u^{2} V\right) V\right)\right\rangle_{L^{2}} \rightarrow\left\langle\chi_{D}, \operatorname{div}\left(\operatorname{div}\left(u^{2} V\right) V\right)\right\rangle_{L^{2}}$, e da equação (5.10) obtemos

$$
\begin{aligned}
\left.\alpha \int_{\Omega}\left(h_{\delta} \circ \varphi_{-t}\right)^{\prime \prime}\right|_{t=0} u^{2} & =\alpha \int_{\Omega} h_{\delta} \operatorname{div}\left(\operatorname{div}\left(u^{2} V\right) V\right) \\
& \rightarrow \alpha \int_{D} \operatorname{div}\left(\operatorname{div}\left(u^{2} V\right) V\right) \\
& =\alpha \int_{\partial D}\left\langle\operatorname{div}\left(u^{2} V\right) V, \nu\right\rangle d S \\
& =\alpha \int_{\partial D} \operatorname{div}\left(u^{2} V\right)\langle V, \nu\rangle d S \\
& =2 \alpha \int_{\partial D} u\langle\nabla u, V\rangle\langle V, \nu\rangle d S+\alpha \int_{\partial D} u^{2} \operatorname{div} V\langle V, \nu\rangle d S
\end{aligned}
$$

Finalmente, substituindo os resultados da equação acima e da equação (5.9) na equação (5.8), 
obtemos

$$
\lambda^{\prime \prime}(0)=2 \alpha \int_{\Gamma} u\left(u^{\prime}+V(u)\right)\langle V, \nu\rangle d S+\alpha \int_{\Gamma} u^{2} \operatorname{div} V\langle V, \nu\rangle d S .
$$

(Vale a mesma observação que fizemos no cálculo da primeira variação: não demonstramos que o limite calculado quando $\delta \rightarrow 0$ é de fato $\lambda^{\prime \prime}(0)$, e também estamos assumindo que $\lambda(t)$ é duas vezes derivável para $t=0)$.

A fórmula de variação acima vale para variações que preservem ou não a medida de $D$. Entretanto, nosso maior interesse está em calcularmos a segunda variação no caso em que a variação $\varphi$ preserva a medida de $D$ e, ainda, quando $D$ é um domínio ótimo para o problema $(*)_{\Omega, \alpha, D}$. Nesse caso, a fórmula da segunda variação é simplificada. Vejamos como. Suponha que a variação $\varphi$ preserva a medida de $D$, isto é, quando $\left|D_{t}\right|=|D| \forall t \in(-\varepsilon, \varepsilon)$. A medida de $D_{t}$ é dada por

$$
\left|D_{t}\right|=\int_{\Omega} \chi_{D_{t}}
$$

e se a medida de $\left|D_{t}\right|$ é constante quando $t$ varia, então

$$
\int_{\Omega} \chi_{D_{t}}^{\prime}=\int_{\Omega} \chi_{D_{t}}^{\prime \prime}=0,
$$

em que o símbolo ' indica a derivação em relação à variável $t$. Usando novamente a aproximação $h_{\delta} \circ \varphi_{-t}$ para a função característica de $D_{t}$ e sendo $\ell_{\delta}=V\left(h_{\delta}\right)$, temos

$$
\begin{aligned}
\left.\int_{\Omega}\left(h_{\delta} \circ \varphi_{-t}\right)^{\prime \prime}\right|_{t=0} & =\int_{\Omega} V\left(\ell_{\delta}\right) \\
& =\int_{\Omega} \operatorname{div}\left(\ell_{\delta} V\right)-\ell_{\delta} \operatorname{div} V \\
& =\int_{\partial \Omega}\left\langle\ell_{\delta} V, \mu\right\rangle d S-\int_{\Omega}\left\langle\nabla h_{\delta},(\operatorname{div} V) V\right\rangle \\
& =-\int_{\Omega}\left\langle\nabla h_{\delta},(\operatorname{div} V) V\right\rangle \\
& =\int_{\Omega} h_{\delta} \operatorname{div}((\operatorname{div} V) V)-\int_{\Omega} \operatorname{div}\left(h_{\delta}(\operatorname{div} V) V\right) \\
& =\int_{\Omega} h_{\delta} \operatorname{div}((\operatorname{div} V) V)-\int_{\partial \Omega} h_{\delta}(\operatorname{div} V)\langle V, \mu\rangle d S \\
& =\int_{\Omega} h_{\delta} \operatorname{div}((\operatorname{div} V) V),
\end{aligned}
$$


pois $V=0$ em $\partial \Omega$ (usamos esse fato duas vezes na equação acima). Quando $\delta \rightarrow 0$,

$$
\int_{\Omega} h_{\delta} \operatorname{div}((\operatorname{div} V) V) \rightarrow \int_{D} \operatorname{div}((\operatorname{div} V) V),
$$

e como a medida de $D_{t}$ é constante devemos ter

$$
\int_{D} \operatorname{div}((\operatorname{div} V) V)=0 \Longleftrightarrow \int_{\Gamma} \operatorname{div} V\langle V, \nu\rangle d S=0 .
$$

Assim, quando a variação preserva a medida de $D$ e $D$ é um domínio ótimo para $(*)_{\Omega, \alpha, D}$, a fórmula

$$
\lambda^{\prime \prime}(0)=2 \alpha \int_{\Gamma} u\left(u^{\prime}+V(u)\right)\langle V, \nu\rangle d S+\alpha \int_{\Gamma} u^{2} \operatorname{div} V\langle V, \nu\rangle d S
$$

se reduz a

$$
\lambda^{\prime \prime}(0)=2 \alpha \int_{\Gamma} u\left(u^{\prime}+V(u)\right)\langle V, \nu\rangle d S,
$$

pois $u$ é constante em $\Gamma$. Obtemos então o seguinte resultado:

Teorema 5.2 (Segunda variação) Se $\varphi$ é uma variação que fixa cada ponto de $\partial \Omega$, então

$$
\lambda^{\prime \prime}(0)=2 \alpha \int_{\Gamma} u\left(u^{\prime}+V(u)\right)\langle V, \nu\rangle d S+\alpha \int_{\Gamma} u^{2} \operatorname{div} V\langle V, \nu\rangle d S
$$

em que $\nu$ é a normal exterior a $\Gamma$, relativa ao conjunto $D$. Além disso, se a variação $\varphi$ preserva a medida de $D$ e $(u, D)$ é um par ótimo, então

$$
\lambda^{\prime \prime}(0)=2 \alpha \int_{\Gamma} u\left(u^{\prime}+V(u)\right)\langle V, \nu\rangle d S .
$$

\subsection{Perspectivas futuras}

O estudo da variação segunda do funcional $D \mapsto \lambda_{\Omega}(\alpha, D)$ leva naturalmente a três tipos de problemas. Primeiro, a estabilidade de uma solução está relacionada ao cálculo da segunda variação. Lembramos que em Cálculo de Variações, estabilidade significa minimalidade local, ou seja, índice de Morse igual a zero. O índice de Morse de um ponto crítico é a dimensão de um espaço maximal de variações do ponto crítico onde a derivada segunda do funcional é negativa.

Um segundo problema a ser estudado, muito interessante, está relacionado com a existência de 
bifurcação e quebra de simetrias das soluções do problema. Em teoria de bifurcação variacional, um resultado conhecido diz que, dada uma família $F_{\alpha}$ a um parâmetro de problemas variacionais e dada uma curva de pontos críticos $x_{\alpha}$, então a existência de um ramo de bifurcação de pontos críticos em $x_{\alpha_{0}}$ é que o índice de Morse de $x_{\alpha}$ tenha um salto em $\alpha_{0}$. Além disso, um resultado famoso de J. Smoller e A. G. Wasserman [21] relaciona bifurcação com quebra de simetrias das soluções. No caso em consideração, o funcional em questão é dado por:

$$
F_{\alpha}(u)=\int_{\Omega}\left(|\nabla u|^{2}+\alpha \chi_{D} u\right) .
$$

Recentemente, R. Pedrosa provou um resultado de quebra de simetria para as soluções do problema da membrana composta. Seria extremamente interessante relacionar esta quebra de simetria com bifurcação, no espírito do trabalho de Smoller e Wasserman.

Um terceiro problema relacionado ao índice de Morse das soluções do problema é o desenvolvimento de uma teoria de Morse. Lembramos que a teoria de Morse fornece estimativas sobre o número de pontos críticos de um funcional não degenerado com um fixado índice de Morse $k$ em termos do k-ésimo número de Betti do domínio do funcional (veja [23]).

Pesquisa nessas direções está sendo realizada em colaboração do Prof. Pedrosa. 


\section{Referências Bibliográficas}

[1] Haïm Brezis. Analyse fonctionelle, théorie et applications. Masson, 1983.

[2] S. Chanillo, D. Grieser, M. Imai, K. Kurata, and I. Ohnishi. Symmetry breaking and other phenomena in the optimization of eigenvalues of composite membranes. Communications of Mathematical Physics, (214):315-337, 2000.

[3] S. Chanillo, D. Grieser, and K. Kurata. The free boundary problem in the optimization of composite membranes. In: Proc. of the Conf. on Geometry and Control, R. Gulliver, W. Litmann, R. Triggiani (eds), Contemporary Math., (268):61-81, 2000.

[4] S. Chanillo and Renato H. L. Pedrosa. Hadamard's formulae for composite membranes.

[5] John B. Conway. A course in functional analysis. Springer-Verlag, 1994.

[6] R. Courant and D. Hilbert. Methods of Mathematical Physics, volume I. Interscience Publishers, New York, 1953.

[7] Djairo Guedes de Figueiredo. Positive solutions of semilinear elliptic problems. Escola LatinoAmericana de Equações Diferenciais, 1981.

[8] Djairo Guedes de Figueiredo. Análise de Fourier e Equações Diferenciais Parciais. Projeto Euclides, IMPA, 2003.

[9] Bruce K. Driver. Analysis tools with applications. Department of Mathematics, University of California, 2003.

[10] C. Faber. Beweiss, dass unter allen homogenen Membrane von gleicher Fläsche und ggleicher Spannung die kreisförmige die tiefsten Grundton gibt. Sitzungsber, Baier, Akad. Math.-Phys. Munich, pages 169-172, 1923. 
[11] Avner Friedman. Foundations of Modern Analysis. Dover Publications, Inc., New York, 1982.

[12] D. Gilbarg and Neil S. Trudinger. Elliptic Partial Differential Equations of Second Order. Springer, 1998.

[13] Enrico Giusti. Minimal surfaces and functions of bounded variation. Birkhäuser, 1984.

[14] E. Krahn. Über eine von Rayleigh formulierte Minmaleigenschaft des Kreises. Math. Ann., (94):97-100, 1925.

[15] Renato H. L. Pedrosa. Some recent results regarding symmetry and symmetry-breaking properties of optimal composite membranes. Progress in Nonlinear Differential Equations and Their Applications, (66):429-442, 2005.

[16] Renato H. L. Pedrosa. Uma Introdução à Simetrização em Análise e Geometria. Publicações Matemáticas, IMPA, 25 Colóquio Brasileiro de Matemática, 2005.

[17] J. W. S. Rayleigh. The Theory of Sound, volume 1, 2. Dover publications, New York, 1945.

[18] Michael Reed and Barry Simon. Functional analysis, volume I of Methods of Modern Mathematical Physics. Academic Press, 1980.

[19] Walter Rudin. Real and Complex Analysis. McGraw-Hill, 1970.

[20] N. Shimakura. Le premiére valeur propre du Laplacien pour le probléme de Dirichlet. J. Math. Pures Appl., (62):129-152, 1983.

[21] J. Smoller and A. G. Wasserman. Bifurcation and symmetry-breaking. Invent. math., (100):6395, 1990.

[22] Guido Stampacchia. Le problème de Dirichlet pour les équations elliptiques du second ordre à coefficients discontinus. Ann. Inst. Fourier, (15):189-258, 1965.

[23] Daniel V. Tausk, Francesco Mercuri, and Paolo Piccione. Notes on Morse theory. With an appendix by Claudio Gorodski. Publicações Matemáticas, IMPA, $23^{\circ}$ Colóquio Brasileiro de Matemática, 2001.

[24] William P. Ziemer. Weakly differentiable functions. Springer-Verlag, 1989. 